\title{
Robust Control of Set-Valued Discrete-Time Dynamical Systems
}

\author{
John S. Baras, Fellow, IEEE, and Nital S. Patel, Member, IEEE
}

\begin{abstract}
This paper presents results obtained for the control of set-valued discrete-time dynamical systems. Such systems model nonlinear systems subject to persistent bounded noise. A robust control problem for such systems is introduced. The problem is formulated as a dynamic game, wherein the controller plays against the set-valued system. Both necessary and sufficient conditions in terms of (stationary) dynamic programming equalities are presented. The output feedback problem is solved using the concept of an information state, where a decoupling between estimation and control is obtained. The methods yield a conceptual approach for constructing controlled-invariant sets and stabilizing controllers for uncertain nonlinear systems.
\end{abstract}

Index Terms - Dynamic games, dynamic inclusions, information state, output feedback, robust control.

\section{INTRODUCTION}

$\mathbf{I}$ $\mathrm{N}$ THIS paper, we consider the robust control of nonlinear systems modeled as inclusions. Examples of systems that give rise to such models include those subject to parametric uncertainty, parameter varying systems (with known bounds on the parameters), and systems subject to bounded disturbances. In the linear systems context, a number of results can be found in the literature concerned with stabilization and ultimate boundedness of systems with uncertain parameters [1]-[5]. Furthermore, in the linear systems context, the problem of systems subject to bounded additive noise is treated under $l_{1}$ optimal control [6], [7]. The inclusion representation employed in this paper enables us to treat these cases in a unified setting. Other systems that one could model in this framework are hybrid systems, where an upper logical level switches between plant models depending on observed events [8]-[10], and systems with discontinuities [11].

In contrast to the large body of results of such problems in the linear context, corresponding results in the nonlinear context are lacking. Furthermore, there is no unifying context within which such problems can be posed. The results presented in this paper contribute toward the development of such a framework. In particular, we show that solving an appropriate robust control problem for inclusions yields controllers that render the closed-loop system ultimately bounded. In certain cases, these results can be considerably strengthened,

Manuscript received November 11, 1994; revised April 25, 1997. Recommended by Associate Editor, C. V. Hollot. This work was supported by the National Science Foundation Engineering Research Centers Program under Grant NSFD CDR 8803012 and the Lockheed Martin Chair in Systems Engineering.

The authors are with the Department of Electrical Engineering and Institute for Systems Research, University of Maryland, College Park, MD 20742 USA. Publisher Item Identifier S 0018-9286(98)00966-0. under smoothness and invariance assumptions, to establish asymptotic stability of the closed-loop system under the absence of exogenous inputs. An important consequence of the approach is that one can conceptually shape the controlledinvariant sets of the closed-loop system. This has immediate bearings on attempts to extend $l_{1}$-optimal control to nonlinear systems [12]. However, unlike the viability theory [13] based approach followed in [7] and [12], our approach yields a dynamic game. It turns out that in this setting, the game involves the controller as one player and the set-valued system as the other. For the output feedback problem, we employ the information state [14], [15] concept to obtain a separation between estimation and control. We furthermore show that under appropriate conditions (certainty equivalence), one can employ an estimate of the state and the state feedback policy. Given that the problem can be cast as a dynamic game, the development parallels the dynamic game framework developed in the nonlinear $H_{\infty}$ context. However, unlike nonlinear $H_{\infty}$, where one is concerned with the $l^{2}$ gain, in the current context we are concerned with asymptotic rejection of bounded disturbances on some regulated output.

In particular, consider the following system:

$$
\begin{aligned}
& x_{k+1}=f\left(x_{k}, u_{k}, w_{k}\right) \\
& y_{k+1}=g\left(x_{k}, u_{k}, v_{k}\right)
\end{aligned}
$$

where $w_{k} \in \mathcal{W}, v_{k} \in \mathcal{V}$, where both $\mathcal{W}$ and $\mathcal{V}$ are bounded sets. The set-valued system is then defined as

$$
\begin{aligned}
& x_{k+1} \in \mathcal{F}\left(x_{k}, u_{k}\right)=\bigcup_{w_{k} \in \mathcal{W}} f\left(x_{k}, u_{k}, w_{k}\right) \\
& y_{k+1} \in \mathcal{G}\left(x_{k}, u_{k}\right)=\bigcup_{v_{k} \in \mathcal{V}} g\left(x_{k}, u_{k}, v_{k}\right)
\end{aligned}
$$

along with a corresponding regulated output of the type

$$
z_{k+1}=l\left(x_{k+1}, u_{k}\right)
$$

where we assume that in general the function $l$ has been obtained via an appropriate selection from a set-valued map. One interpretation of our results is, given $\gamma>0$, one is trying to obtain a controller such that if $x_{0}=0$, then $\left|\tilde{z}_{k+1}\right| \leq \gamma$, for all $k \geq 0$; else, if $x_{0} \neq 0$, then limsup $\sup _{k \rightarrow \infty}\left|\tilde{z}_{k+1}\right| \leq \gamma$. Here $\tilde{z}_{k+1}=h\left(x_{k+1}, u_{k}\right)$, with $h$ equal to the partial derivative of $l$ with respect to the states $(x)$. However, for the sake of generality, we will set up and study the problem for inclusions. We will present both necessary and sufficient conditions for solvability, along with a version of the bounded real lemma. The latter is stated in terms of a dissipation inequality and has 
appeared repeatedly in papers dealing with nonlinear robust control (e.g., [14] and [16]-[20]).

The rest of the paper is organized as follows. In Section II, we formulate the robust control problem for inclusions. Section III deals with the state feedback case, followed by Section IV which treats the output feedback case. Certainty equivalence is discussed in Section $\mathrm{V}$, and an example is presented in Section VI.

\section{PROBLEM Formulation}

The system under consideration $(\Sigma)$ is expressed as

$$
\Sigma \begin{cases}x_{k+1} \in \mathcal{F}\left(x_{k}, u_{k}\right), & x_{0} \in X_{0} \\ y_{k+1} \in \mathcal{G}\left(x_{k}, u_{k}\right) & \\ z_{k+1}=l\left(x_{k+1}, u_{k}\right), & k=0,1, \cdots .\end{cases}
$$

Here, $x_{k} \in \boldsymbol{R}^{n}$ are the states, with $X_{0}$ denoting the set of possible initial state values, $u_{k} \in \boldsymbol{U} \subset \boldsymbol{R}^{m}$ are the control inputs, $y_{k} \in \boldsymbol{R}^{t}$ are the measured variables, and $z_{k} \in \boldsymbol{R}^{q}$ are the regulated outputs. We will employ the following notation throughout the paper.

| $\mid$ denotes any suitable norm.

$x_{i, j}$ denotes a sequence $\left\{x_{i}, x_{i+1}, \cdots, x_{j}\right\}$.

$\Gamma_{0, k}^{u}(x)$ denotes the truncated forward cone of the point $x \in \boldsymbol{R}^{n}$ [21]. In particular

$$
\begin{aligned}
\Gamma_{0, k}^{u}(x) \triangleq & \left\{x_{0, k} \mid x_{j+1} \in \mathcal{F}\left(x_{j}, u_{j}\right),\right. \\
& \left.j=0, \cdots, k-1 ; x_{0}=x\right\}
\end{aligned}
$$

i.e., $\Gamma_{0, k}^{u}(x)$ is the set of all possible state trajectories that the system can generate in the time interval $[0, k]$, given a control policy $u$ and initial condition $x$. In case the time horizon under consideration is infinite, we simply write $\Gamma^{u}(x)$.

$\mathcal{X}_{k}^{u}\left(x_{0}\right) \subset \boldsymbol{R}^{n}$ is the cross section of the forward cone of $x_{0}$ at time instant $k$. In particular, it represents the set of states that the system could be in at time $k$, under a policy $u$, with the initial state equal to $x_{0}$.

We furthermore write $r, s \in \Gamma_{0, k}^{u}(x)$ to denote the set of trajectories such that $r \in \Gamma_{0, k}^{u}(x)$, and $s_{i+1} \in \mathcal{F}\left(r_{i}, u_{i}\right)$ for $i=0, \cdots, k-1, r, s \in \Gamma^{u}(x)$ is defined similarly.

$\mathcal{B}_{a}(b)$ denotes an open ball of radius $a$ centered at $b$, and $\overline{\mathcal{B}}_{a}(b)$ similarly denotes a closed ball.

$S$ and $O$ denote the space of static state and dynamic output feedback policies respectively. If $\bar{u} \in S$, then for any $k$, the control value $u_{k}=\bar{u}\left(x_{k}\right) \in \boldsymbol{U}$. Furthermore, we write $S_{i, j}$ to denote policies defined only for the time interval $i, i+1, \cdots, j$. Similarly, if $\bar{u} \in O$, then for any $k, u_{k}=\bar{u}\left(y_{1}, k\right) \in \boldsymbol{U}$. Furthermore, $O_{i, j}$ is defined in a similar manner as $S_{i, j}$.

$\delta_{M}: \boldsymbol{R}^{n} \rightarrow \boldsymbol{R}^{*}$ is defined by

$$
\delta_{M}(x) \triangleq \begin{cases}0, & \text { if } x \in M \\ -\infty, & \text { else. }\end{cases}
$$

For the output feedback case, we define

$$
\begin{aligned}
\Delta_{1, K}^{u}\left(x_{0}\right)= & \left\{y_{1, K} \mid y_{k+1} \in \mathcal{G}\left(x_{k}, u_{k}\right),\right. \\
& \left.\forall x \in \Gamma_{0, K-1}^{u}\left(x_{0}\right)\right\} \\
\Gamma_{0, K}^{u, y}\left(x_{0}\right)= & \left\{x_{0, K} \in \Gamma_{0, K}^{u}\left(x_{0}\right) \mid y_{k+1} \in \mathcal{G}\left(x_{k}, u_{k}\right),\right. \\
& k=0, \cdots, K-1\} .
\end{aligned}
$$

We will also write $r, s \in \Gamma_{0, K}^{u, y}(x)$ in a similar manner as for $\Gamma_{0, K}^{u}(x)$. Lastly, $\Delta^{u}\left(x_{0}\right)$ and $\Gamma^{u, y}\left(x_{0}\right)$ represent the infinite horizon cases [as in $\Gamma^{u}\left(x_{0}\right)$ ].

Given any set-valued map $\Lambda(x)$, we occasionally write

$$
\Lambda(M)=\bigcup_{x \in M} \Lambda(x) .
$$

Finally, $\Sigma_{x}^{u}$ denotes the system with an initial state value $x$, employing a control policy $u$, and $\Sigma^{u}$, the system under control policy $u$ and any initial state value $x_{0} \in X_{0}$.

Remark 1: Of particular interest here is the case when $|\cdot|$ denotes the $\infty$ norm. But since the results are normindependent, we choose to pursue this level of generality.

The following assumptions are made on the system $\Sigma$.

A1) $X_{0}$ is closed, and $0 \in X_{0}$.

A2) $\mathcal{F}(x, u), \mathcal{G}(x, u)$ are compact for all $x \in \boldsymbol{R}^{n}$ and $u \in U$.

A3) The origin is an equilibrium point for $\mathcal{F}, \mathcal{G}$, and $l$, i.e.,

$$
\mathcal{F}(0,0) \ni 0, \quad \mathcal{G}(0,0) \ni 0, \quad l(0,0)=0 .
$$

A4) For any $\epsilon>0$, for any $x \in \boldsymbol{R}^{n}, u \in \boldsymbol{U}$, and any $r \in \mathcal{F}(x, u)$, there exists $\epsilon_{2}>0$ such that $\mathcal{B}_{\epsilon_{2}}(s) \subset$ $\mathcal{B}_{\epsilon}(r) \bigcap \mathcal{F}(x, u)$ for some $s \in \mathcal{B}_{\epsilon}(r) \bigcap \mathcal{F}(x, u)$.

A5) $l(\cdot, u) \in C^{1}\left(\boldsymbol{R}^{n}\right)$ for all $u \in \boldsymbol{U}$ and is such that $\exists \gamma \geq 0$, such that

$$
\mathcal{L}^{\gamma} \triangleq\left\{s \in \boldsymbol{R}^{n} \mid \exists u \in \boldsymbol{U} \text { s.t. }\left|\frac{\partial}{\partial x} l(s, u)\right| \leq \gamma\right\}
$$

is compact and contains the origin $\forall \gamma>\gamma$. In particular, $\gamma$ represents the smallest value of $\bar{\gamma}$ for which $0 \in \mathcal{L}^{\gamma}$. In what follows, without loss of generality, we assume that $\gamma=0$.

A6) $\boldsymbol{U} \subset \boldsymbol{R}^{m}$ is compact.

Remark 2: The smoothness assumptions in A5) can be replaced by directional differentiability. Also in A5), we can replace compactness by boundedness. Furthermore, in A5) we can get away with a subset of the states being bounded, provided we can use invariance to establish that the remaining states are bounded as well. We can also relax A4), to let $\mathcal{F}$ be locally connected. How one deals with these situations is very much problem dependent, and for clarity we assume that the above assumptions hold.

The robust control problem can now be stated as: Given $\gamma>0$, find a controller $u(\in S$ or $O$ depending on what is measured) such that the closed-loop system $\Sigma^{u}$ satisfies the following three conditions.

C1) $\Sigma^{u}$ is weakly asymptotically stable, in the sense that for each $k$, there exists an $\alpha_{k} \in \mathcal{F}\left(x_{k}, u_{k}\right)$ such that the sequence $\alpha_{k} \rightarrow 0$ as $k \rightarrow \infty$.

C2) $\Sigma^{u}$ is ultimately bounded, i.e., there exists a bounded set $\mathcal{M}$ such that for any state trajectory $\left\{x_{k}\right\}$ one has $x_{k} \rightarrow \mathcal{M}$ as $k \rightarrow \infty$.

C3) (Finite Gain) Given a $q \in[1, \infty)$ there exists a finite $\beta^{u}(x)$, with $\beta^{u}(0)=0$ such that

$$
\begin{aligned}
& \sup _{r, s \in \Gamma^{u}\left(x_{0}\right)} \sum_{i=0}^{\infty}\left|l\left(r_{i+1}, u_{i}\right)-l\left(s_{i+1}, u_{i}\right)\right|^{q} \\
& \quad-\gamma^{q}\left|r_{i+1}-s_{i+1}\right|^{q} \leq \beta^{u}\left(x_{0}\right), \quad \forall x_{0} \in X_{0} .
\end{aligned}
$$




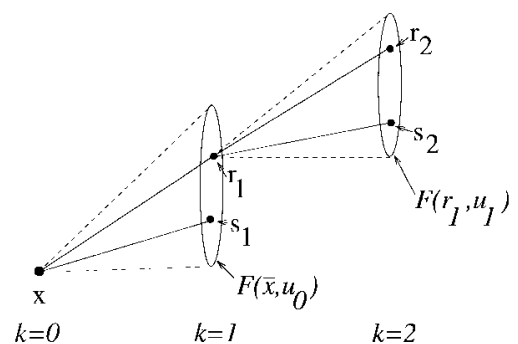

Fig. 1. Set of possible state trajectories.

We call the closed-loop system finite gain if C3) is satisfied.

The cost employed in C3) can be interpreted as setting up the robust control problem so as to attenuate the influence of the set-valued dynamics on the regulated output $z$. To this end consider a finite-time problem, where the time horizon is two. We are given a $\gamma>0$ and an admissible control policy $u$. We denote the initial state value by $\bar{x}$. Consider Fig. 1 . From $\bar{x}$, the system can go to any point in $\mathcal{F}\left(\bar{x}, u_{0}\right)$. Suppose that the next state the system goes to is $r_{1}$. Note that the system could have also gone to $s_{1}$. Now, from $r_{1}$ we can go to any arbitrary point in $\mathcal{F}\left(r_{1}, u_{1}\right)$, where $u_{1}$ is the control value at time $k=1$. We again pick two points $r_{2}, s_{2}$ in $\mathcal{F}\left(r_{1}, u_{1}\right)$. The variation in the regulated output that could occur is therefore

$$
\left(\left|l\left(r_{1}, u_{0}\right)-l\left(s_{1}, u_{0}\right)\right|^{q}+\left|l\left(r_{2}, u_{1}\right)-l\left(s_{2}, u_{1}\right)\right|^{q}\right)^{1 / q} .
$$

We now normalize this by the distance between $r$ and $s$, i.e., by

$$
\left(\left|r_{1}-s_{1}\right|^{q}+\left|r_{2}-s_{2}\right|^{q}\right)^{1 / q} .
$$

The reason for doing so is that we are trying to attenuate the influence of the set-valued dynamics on the regulated output and not the variation in the regulated output itself. We can write the worst case normalized variation in the regulated output as

$$
\begin{aligned}
& \sup _{r, s \in \Gamma_{0,2}^{u}(\bar{x})} \\
& \quad \frac{\left(\left|l\left(r_{1}, u_{0}\right)-l\left(s_{1}, u_{0}\right)\right|^{q}+\left|l\left(r_{2}, u_{1}\right)-l\left(s_{2}, u_{1}\right)\right|^{q}\right)^{1 / q}}{\left(\left|r_{1}-s_{1}\right|^{q}+\left|r_{2}-s_{2}\right|^{q}\right)^{1 / q}} .
\end{aligned}
$$

If $\bar{x}=0$, we now require that for the given $\gamma$, the control policy must be such that this worst case normalized variation is bounded by $\gamma$ or that

$$
\begin{aligned}
& \sup _{r, s \in \Gamma_{0,2}^{u}(0)} \sum_{k=1}^{2} \mid l\left(r_{k}, u_{k-1}\right) \\
& \quad-\left.l\left(s_{k}, u_{k-1}\right)\right|^{q}-\gamma^{q}\left|r_{k}-s_{k}\right|^{q} \leq 0 .
\end{aligned}
$$

Generalizing to arbitrary $\bar{x} \in X_{0}$, we require the existence of a finite $\beta^{u}(x) \geq 0, \beta^{u}(0)=0$, such that

$$
\begin{aligned}
& \sup _{r, s \in \Gamma_{0,2}^{u}(\bar{x})} \sum_{k=1}^{2}\left|l\left(r_{k}, u_{k-1}\right)-l\left(s_{k}, u_{k-1}\right)\right|^{q} \\
& \quad-\gamma^{q}\left|r_{k}-s_{k}\right|^{q} \leq \beta^{u}(\bar{x})
\end{aligned}
$$

for all $\bar{x} \in X_{0}$. This condition requires that the worst case normalized variation of the regulated output be finite for all initial conditions. One now repeats the above process for an arbitrary large time horizon to obtain (3).
Remark 3: Assumption A4) precludes $\mathcal{F}(x, u)$ from being a singleton, for which C3) is trivially satisfied. Assumption A5) precludes certain types of cost functions, e.g., linear, for which C3) will be trivialized by $\gamma$ large enough.

\section{State Feedback CASE}

In the state feedback case, the problem is to find a controller $u \in S$, i.e., $u_{k}=u\left(x_{k}\right)$, where $u: \boldsymbol{R}^{n} \longmapsto \boldsymbol{U}$, such that the three conditions stated above are satisfied. For the state feedback case, we could have considered a more general problem, with state dependent control constraints $\boldsymbol{U}(x)$. However, the development of this section is unaffected.

\section{A. Finite-Time Case}

For the finite-time case, conditions $\mathrm{C} 1$ ) and $\mathrm{C} 2$ ) are not required. From C3) we require for a given $K>0$, the existence of a finite $\beta_{K}^{u}\left(x_{0}\right), \beta_{K}^{u}(0)=0$ such that

$$
\begin{aligned}
& \sum_{i=0}^{K-1}\left(\left|l\left(r_{i+1}, u_{i}\right)-l\left(s_{i+1}, u_{i}\right)\right|^{q}-\gamma^{q}\left|r_{i+1}-s_{i+1}\right|^{q}\right) \\
& \quad \leq \beta_{K}^{u}\left(x_{0}\right), \quad \forall r, s \in \Gamma_{0, K}^{u}\left(x_{0}\right), \quad \forall x_{0} \in X_{0} .
\end{aligned}
$$

1) Dynamic Game: Here, the robust control problem is converted into an equivalent dynamic game. For $u \in S_{k, K-1}$ and $\bar{x} \in \mathcal{X}_{k}^{u}\left(x_{0}\right)$, for any $x_{0} \in X_{0}$, define

$$
\begin{aligned}
J_{\bar{x}, k}(u)=\sup _{r, s \in \Gamma_{k, K}^{u}(\bar{x})} & \left\{\sum _ { i = k } ^ { K - 1 } \left(\left|l\left(r_{i+1}, u_{i}\right)-l\left(s_{i+1}, u_{i}\right)\right|^{q}\right.\right. \\
& \left.\left.-\gamma^{q}\left|r_{i+1}-s_{i+1}\right|^{q}\right)\right\}
\end{aligned}
$$

Clearly

$$
J_{\bar{x}, k}(u) \geq 0 .
$$

Now, the finite-gain property can be expressed as below.

Lemma 1: $\Sigma \bar{u}$ is a finite gain on $[k, K]$ if and only if there exists a finite $\beta_{K}^{u}(\bar{x}), \beta_{K}^{u}(0)=0$ such that

$$
J_{\bar{x}, j}(u) \leq \beta_{K}^{u}(\bar{x}), \quad j \in[k, K], \quad \forall \bar{x} \in X_{0} .
$$

The problem is hence reduced to finding a $u^{*} \in S_{k, K-1}$ which minimizes $J_{\bar{x}, k}$.

2) Solution to the Finite-Time State Feedback Robust Control Problem: We can solve the above problem using dynamic programming. Define

$$
\begin{gathered}
V_{k}(\bar{x})=\inf _{u \in S_{k, K-1}} \sup _{r, s \in \Gamma_{k, K}^{u}(\bar{x})}\left\{\sum_{i=k}^{K-1}\left|l\left(r_{i+1}, u_{i}\right)-l\left(s_{i+1}, u_{i}\right)\right|^{q}\right. \\
\left.-\gamma^{q}\left|r_{i+1}-s_{i+1}\right|^{q}\right\} . \\
\text { The corresponding dynamic programming equation is } \\
\begin{aligned}
V_{k}(x)=\inf _{u \in \boldsymbol{U}} \sup _{r, s \in \mathcal{F}(x, u)}\left\{|l(r, u)-l(s, u)|^{q}-\gamma^{q}|r-s|^{q}\right. \\
\left.+V_{k+1}(r)\right\} \\
V_{K}(x)=0 .
\end{aligned}
\end{gathered}
$$


Note that we have abused notation, and here $u$ is a vector instead of a function as in (5).

Theorem 1 (Necessity): Assume that $u^{*} \in S_{0, K-1}$ solves the finite-time state feedback robust control problem. Then, there exists a solution $V$ to the dynamic programming equation (8) such that $V_{k}(x) \geq 0, V_{k}(0)=0, k \in[0, K-1], x \in X_{0}$.

Proof: For $x \in X_{0}, k \in[0, K-1]$ define

$$
V_{k}(x)=\inf _{u \in S_{k, K-1}} J_{x, k}(u) .
$$

Then, we have

$$
0 \leq V_{k}(x) \leq \beta_{K}^{u^{*}}(x), \quad k \in[0, K-1], \quad x \in X_{0} .
$$

Thus, $V_{k}$ is finite on $X_{0}$, and by dynamic programming, $V$ satisfies (8). Also, since $\beta_{K}^{u^{*}}(0)=0, V_{k}(0)=0$.

Theorem 2 (Sufficiency): Assume that there exists a solution $V$ to the dynamic programming equation (8), such that $V_{k}(x) \geq 0, V_{k}(0)=0, k \in[0, K-1], x \in X_{0}$. Let $u^{*} \in S_{k, K-1}$ be a control policy such that $u_{k}^{*}$ achieves the minimum in (8) for $k=0, \cdots, K-1$. Then $u^{*}$ solves the finite-time state feedback robust control problem.

Proof: Dynamic programming arguments imply that for a given $x \in X_{0}$

$$
V_{0}(x)=J_{x, 0}\left(u^{*}\right)=\inf _{u \in S_{0}, K-1} J_{x, 0}(u) .
$$

Thus $u^{*}$ is an optimal policy for the game and Lemma 1 is satisfied with $u=u^{*}$, where we obtain $\beta_{K}^{u}(x)=V_{0}(x)$.

\section{B. Infinite-Time Case}

Here, we are interested in the limit as $K \rightarrow \infty$. Invoking stationarity (8) becomes

$$
\begin{gathered}
V(x)=\inf _{u \in \boldsymbol{U}} \sup _{r, s \in \mathcal{F}(x, u)}\left\{V(s)+|l(r, u)-l(s, u)|^{q}\right. \\
\left.-\gamma^{q}|r-s|^{q}\right\} .
\end{gathered}
$$

1) The Dissipation Inequality: We say that the system $\Sigma^{\bar{u}}$ is finite-gain dissipative if there exists a function $V(x)$ (called the storage function) such that $V(x) \geq 0, V(0)=0$, and it satisfies the dissipation inequality

$$
\begin{aligned}
V(x) \geq \sup _{r, s \in \mathcal{F}(x, \bar{u}(x))}\{ & V(s)-\gamma^{q}|r-s|^{q}+\mid l(r, \bar{u}(x)) \\
& \left.-\left.l(s, \bar{u}(x))\right|^{q}\right\}, \quad \forall x \in \mathcal{X}_{k}^{\bar{u}}\left(x_{0}\right), \\
& \forall k \geq 0, \quad \forall x_{0} \in X_{0}
\end{aligned}
$$

where $\bar{u}(x)$ is the control value for state $x$.

Theorem 3: Let $u \in S$. The system $\Sigma^{u}$ is finite gain if and only if it is finite-gain dissipative.

Proof $i$ ): Assume $\Sigma^{u}$ is finite-gain dissipative. Then (10) implies

$$
\begin{aligned}
V\left(x_{0}\right) \geq & V\left(r_{k}\right)+\sum_{i=0}^{k-1}\left|l\left(r_{i+1}, u_{i}\right)-l\left(s_{i+1}, u_{i}\right)\right|^{q} \\
& \quad-\gamma^{q}\left|r_{i+1}-s_{i+1}\right|^{q}, \quad \forall k>0 ; \quad \forall r, s \in \Gamma^{u}\left(x_{0}\right) .
\end{aligned}
$$

Since $V \geq 0$ for all $r, s \in \Gamma_{0, k}^{u}\left(x_{0}\right)$, this implies

$$
\sum_{i=0}^{k-1}\left|l\left(r_{i+1}, u_{i}\right)-l\left(s_{i+1}, u_{i}\right)\right|^{q}-\gamma^{q}\left|r_{i+1}-s_{i+1}\right|^{q} \leq V\left(x_{0}\right) \text {. }
$$

Thus $\Sigma^{u}$ is finite gain. ii): Assume $\Sigma^{u}$ is finite gain. For any $x_{0} \in X_{0}$ and $k \geq 0$, define for $x \in \mathcal{X}_{k}^{u}\left(x_{0}\right)$

$$
\begin{aligned}
\tilde{V}_{k, j}^{u}\left(x, x_{0}\right)=\sup _{r, s \in \Gamma^{u}(x)} & \left\{\sum_{i=0}^{j-1}\left|l\left(r_{i+1}, u_{i}\right)-l\left(s_{i+1}, u_{i}\right)\right|^{q}\right. \\
& \left.-\gamma^{q}\left|r_{i+1}-s_{i+1}\right|^{q}\right\} .
\end{aligned}
$$

Then we have for any $x \in \mathcal{X}_{k}^{u}\left(x_{0}\right)$

$$
0 \leq \tilde{V}_{k, j}^{u}\left(x, x_{0}\right) \leq \beta^{u}\left(x_{0}\right), \quad \forall j \geq 0
$$

and

$$
\tilde{V}_{k, j+1}^{u}\left(x, x_{0}\right) \geq \tilde{V}_{k, j}^{u}\left(x, x_{0}\right), \quad \forall x \in \mathcal{X}_{k}^{u}\left(x_{0}\right) .
$$

Furthermore, note that by time invariance, $\tilde{V}_{k, j}^{u}\left(x, x_{0}\right)$ depends only on $x$ and $j$. Thus if $x \in \mathcal{X}_{k_{1}}^{u}\left(x_{0}^{1}\right) \bigcap \mathcal{X}_{k_{2}}^{u}\left(x_{0}^{2}\right)$, then $\tilde{V}_{k_{1}, j}^{u}\left(x, x_{0}^{1}\right) \equiv \tilde{V}_{k_{2}, j}^{u}\left(x, x_{0}^{2}\right)$. Hence

$$
\begin{gathered}
\tilde{V}_{k, j}^{u}\left(x, x_{0}\right) \rightarrow V^{u}(x), \quad \text { as } \quad k \rightarrow \infty, \quad \forall x \in \mathcal{X}_{k}^{u}\left(x_{0}\right), \\
\quad k \geq 0, x_{0} \in X_{0} .
\end{gathered}
$$

Also, we have

$$
0 \leq V^{u}\left(x_{0}\right) \leq \beta^{u}\left(x_{0}\right)
$$

Since

$$
V(x)=\inf _{u \in S} V^{u}(x)=V^{\bar{u}}(x) \leq V^{u}(x)
$$

dynamic programming implies that $V^{u}(x)$ solves the dissipation inequality (10) for all $x \in \mathcal{X}_{k}^{u}\left(x_{0}\right), k \geq 0, x_{0} \in X_{0}$. Furthermore, $V^{u}(x) \geq 0$ and $V^{u}(0)=0$. Thus $V^{u}$ is a storage function and hence $\Sigma^{u}$ is finite-gain dissipative.

We now have to show that the control policy $u \in S$, which renders $\Sigma^{u}$ finite-gain dissipative, also guarantees ultimate boundedness of trajectories; furthermore, under a certain detectability type assumption, we have the existence of a sequence $\alpha_{n} \in \mathcal{F}\left(x_{n}, u_{n}\right)$ such that $\lim _{n \rightarrow \infty} \alpha_{n}=0$. The above can be also expressed as [22]

$$
0 \in \liminf _{k \rightarrow \infty} \mathcal{F}\left(x_{k}, u_{k}\right) .
$$

Before proceeding further, we place an additional assumption on the system $\Sigma$.

A7) Assume that for a given $\gamma>0$, the system $\Sigma^{\bar{u}}$ is such that

$$
\limsup _{k \rightarrow \infty}\left|\frac{\partial}{\partial x} l\left(\bar{x}_{k+1}, \bar{u}_{k}\right)\right| \leq \gamma
$$

implies $0 \in \liminf _{k \rightarrow \infty} \mathcal{F}\left(\bar{x}_{k}, \bar{u}_{k}\right)$.

Remark 4: The assumption above can be viewed to be analogous to the detectability assumption often encountered in $H_{\infty}$ control literature, e.g., [18] and [19]. It also represents a tightness condition for $\gamma$.

The following theorem gives a sufficient condition for weak asymptotic stability.

Theorem 4: If for a given $\gamma>0, \Sigma^{\bar{u}}$ is finite-gain dissipative and satisfies Assumption A7), then $\Sigma^{\bar{u}}$ is weakly asymptotically stable. 
Proof: From the dissipation inequality (10), we obtain for any $x_{0} \in X_{0}$

$$
\begin{aligned}
& \sum_{i=0}^{K}\left|l\left(r_{i+1}, \bar{u}_{i}\right)-l\left(s_{i+1}, \bar{u}_{i}\right)\right|^{q} \\
& \quad-\gamma^{q}\left|r_{i+1}-s_{i+1}\right|^{q} \leq V\left(x_{0}\right), \quad \forall K ; r, s \in \Gamma^{\bar{u}}\left(x_{0}\right) .
\end{aligned}
$$

In particular for any $x \in \Gamma^{\bar{u}}\left(x_{0}\right)$

$$
\sum_{k=0}^{K} W_{k}^{\bar{u}} \leq V\left(x_{0}\right), \quad \forall K
$$

where $W_{k}^{\bar{u}}$ is defined in (25). We know that $W_{k}^{\bar{u}} \geq 0, \forall k$. This implies that

$$
W_{k}^{\bar{u}} \rightarrow 0 \quad \text { as } \quad k \rightarrow \infty .
$$

Hence, by Corollary 6 and Assumption A7) we obtain

$$
0 \in \liminf _{k \rightarrow \infty} \mathcal{F}\left(\bar{x}_{k}, \bar{u}_{k}\right) .
$$

This implies that $\exists \alpha_{n} \in \mathcal{F}\left(\bar{x}_{n}, \bar{u}_{n}\right)$ such that $\lim _{n \rightarrow \infty} \alpha_{n}=$ 0. Hence, $\forall x \in \Gamma^{\bar{u}}\left(x_{0}\right), \exists \alpha_{n} \in \mathcal{F}\left(x_{n}, \bar{u}_{n}\right)$ such that $\lim _{n \rightarrow \infty} \alpha_{n}=0$.

Corollary 1: If $\Sigma^{\bar{u}}$ is finite-gain dissipative, then $\Sigma^{\bar{u}}$ is ultimately bounded.

Proof: In the proof of Theorem 4, we observe that if $\Sigma^{\bar{u}}$ is finite-gain dissipative, then

$$
W_{k}^{\bar{u}} \rightarrow 0 \quad \text { as } \quad k \rightarrow \infty .
$$

Hence, by Corollary 6

$$
\limsup _{k \rightarrow \infty}\left|\frac{\partial}{\partial x} l\left(x_{k+1}, \bar{u}_{k}\right)\right| \leq \gamma
$$

which implies that as $k \rightarrow \infty, x_{k} \rightarrow \mathcal{L}^{\gamma}$, which is bounded by Assumption A5). Then there exists an $\epsilon>0$, s.t. $\forall K \geq$ $0, \exists k \geq K$, such that $\mathcal{B}_{\epsilon}\left(x_{k}\right) \bigcap \mathcal{L}^{\gamma}=\phi$. This implies that there exists an $\hat{\epsilon}>0$, such that $\forall K \geq 0, \exists k \geq K$, such that $\left|(\partial / \partial x) l\left(x_{k+1}, \bar{u}_{k}\right)\right|>\gamma+\hat{\epsilon}$ which implies that

$$
\limsup _{k \rightarrow \infty}\left|\frac{\partial}{\partial x} l\left(x_{k+1}, \bar{u}_{k}\right)\right|>\gamma
$$

and here we have a contradiction. In particular, due to the continuity of $(\partial / \partial x) l(x, u)$ and compactness of $\mathcal{L}^{\gamma}$, the two conditions are equivalent, i.e.,

$$
\limsup _{k \rightarrow \infty}\left|\frac{\partial}{\partial x} l\left(x_{k+1}, \bar{u}_{k}\right)\right| \leq \gamma \text { iff } x_{k} \rightarrow \mathcal{L}^{\gamma} \text {, as } k \rightarrow \infty .
$$

Remark 5: If we impose sufficient smoothness assumptions on $\Sigma^{\bar{u}}$, such that $V$ is continuous in (10), then all trajectories generated by $\Sigma^{\bar{u}}$ are stable in the sense of Lyapunov, with $V$ the corresponding Lyapunov function. In this context, the work of Blanchini [23] is similar in spirit, where state feedback compensators were constructed for discrete-time linear systems to achieve ultimate boundedness control via setinduced Lyapunov functions. This procedure was then applied to the state feedback $l_{1}$-optimal control problem [24].
Remark 6: It is clear from above and from Lemma 8 that we do need some form of continuity assumption on $l$ as a necessary condition for the system to be finite-gain dissipative.

2) Solution to the State Feedback Robust Control Problem: Although the results above indicate that the controlled dissipation inequality is both a necessary and sufficient condition for the solvability of the state feedback robust control problem, we state here the necessary and sufficient conditions in terms of dynamic programming equalities.

Theorem 5 (Necessity): If a controller $\bar{u} \in S$ solves the state feedback robust control problem, then there exists a function $V(x)$ such that $V(x) \geq 0, V(0)=0$ and $V$ satisfies the following equation, i.e.:

$$
\begin{aligned}
V(x)=\inf _{u \in \boldsymbol{U}} \sup _{r, s \in \mathcal{F}(x, u)}\left\{|l(r, u)-l(s, u)|^{q}\right. \\
\left.-\gamma^{q}|r-s|^{q}+V(r)\right\}
\end{aligned}
$$

$x \in \mathcal{X}_{k}^{\bar{u}}\left(x_{0}\right), k \geq 0, x_{0} \in X_{0}$.

Proof: Construct a sequence $V_{j}, j=0, \cdots$ of functions as follows:

$$
\begin{aligned}
V_{j+1}(x) & =\inf _{u \in \boldsymbol{U}} \sup _{r, s \in \mathcal{F}(x, u)}\left\{|l(r, u)-l(s, u)|^{q}\right. \\
& \left.-\gamma^{q}|r-s|^{q}+V_{j}(r)\right\} \\
V_{0}(x) & =0 .
\end{aligned}
$$

Clearly

$$
V_{j}(x) \geq 0, \quad \forall x \in \operatorname{Re}^{n}, \quad \forall j \geq 0
$$

and

$$
V_{j+1}(x) \geq V_{j}(x), \quad \forall x \in \operatorname{Re}^{n}, \quad j \geq 0 .
$$

For any $x_{0} \in X_{0}$ and $k \geq 0$, pick an $x \in \mathcal{X}_{k}^{\bar{u}}\left(x_{0}\right)$. Then dynamic programming arguments imply that

$$
0 \leq V_{j}(x) \leq \beta^{\bar{u}}\left(x_{0}\right), \quad \forall x \in \mathcal{X}_{k}^{\bar{u}}\left(x_{0}\right) .
$$

Furthermore, note that $V_{j}(x)$ depends only on $j$ and $x$. Hence

$$
\begin{gathered}
V_{j}(x) \rightarrow V(x) \text { as } j \rightarrow \infty, \quad \forall x \in \mathcal{X}_{k}^{\bar{u}}\left(x_{0}\right) \\
k \geq 0, \quad x_{0} \in X_{0}
\end{gathered}
$$

and by definition, $V$ satisfies (11). Furthermore, $V(x) \geq 0$ and $V\left(x_{0}\right) \leq \beta^{\bar{u}}\left(x_{0}\right)$. Hence, $V(0)=0$.

Theorem 6 (Sufficiency): Assume that there exists a solution $V$ to the stationary dynamic programming equation (11) for all $x \in \mathcal{D} \subset \boldsymbol{R}^{n}, 0 \in \mathcal{D}$, satisfying $V(x) \geq 0, x \in \mathcal{D}$, and $V(0)=0$. Let $\bar{u}(x)$ be the control value which achieves the minimum in (11). Then $\bar{u} \in S$ solves the state feedback robust control problem provided that $\Sigma^{\bar{u}}$ satisfies assumption A7) and $\bigcup_{x_{0} \in X_{0}} \bigcup_{k>0} \mathcal{X}_{k}^{\bar{u}}\left(x_{0}\right) \subset \mathcal{D}$.

Proof: Since $V$ satisfies (11), $\Sigma^{\bar{u}}$ satisfies (10) with equality. Hence, $\Sigma^{\bar{u}}$ is finite-gain dissipative, and hence by Theorem $3 \Sigma^{\bar{u}}$ is finite gain. Furthermore, by Theorem $4 \Sigma^{\bar{u}}$ is weakly asymptotically stable, and by Corollary $1 \Sigma^{\bar{u}}$ is ultimately bounded. 
3) Characterization of Controlled-Invariant Sets: For a state feedback policy $u \in S$, let $\mathcal{K}_{S}^{u}$ be defined as that set for which: 1) $0 \in \mathcal{K}_{s}^{u}$ and 2) if $x_{0} \in \mathcal{K}_{S}^{u}$, then $x_{k} \in \mathcal{K}_{S}^{u}$ for all $k \geq 0$. We call such a set $\mathcal{K}_{S}^{u}$ controlled-invariant with respect to policy $u$. The solution $\bar{u} \in S$ to the robust control problem guarantees that the system $\Sigma^{\bar{u}}$ is ultimately bounded. In particular, we had for any state trajectory $x \in \Gamma^{\bar{u}}\left(X_{0}\right), x_{k} \rightarrow \mathcal{L}^{\gamma}$, as $k \rightarrow \infty$. We now show that there exists a set $\mathcal{K}_{S}^{\bar{u}} \subset \mathcal{L}^{\gamma}$, such that $\mathcal{K}_{S}^{\bar{u}}$ is a controlledinvariant set for the closed-loop system. Hence, any state trajectory starting in $\mathcal{K}_{S}^{\bar{u}}$ remains in $\mathcal{K}_{S}^{\bar{u}}$.

Theorem 7 (Sufficiency): Suppose conditions of Theorem 6 are satisfied, with $\bar{u} \in S$ the resulting policy. Then there exists a $\mathcal{K}_{S}^{\bar{u}} \subset \mathcal{L}^{\gamma}$ such that i) $0 \in \mathcal{K}_{S}^{\bar{u}}$ ii) furthermore, if $x_{0} \notin \mathcal{K}_{S}^{\bar{u}}$, then $x_{k} \rightarrow \mathcal{L}^{\gamma}$ as $k \rightarrow \infty$.

Proof: To establish part i), suppose the system has initial condition $x_{0}=0$. By A5), $0 \in \mathcal{L}^{\gamma}$. Since $V(0)=0$, this implies that for any $r \in \Gamma^{\bar{u}}(0)$, for any $k \geq 0$, and any $s_{k+1} \in \mathcal{F}\left(r_{k}, \bar{u}_{k}\right)$, with $s_{k+1} \neq r_{k+1}$, we have

$$
\left|l\left(r_{k+1}, \bar{u}_{k}\right)-l\left(s_{k+1}, \bar{u}_{k}\right)\right|^{q}-\gamma^{q}\left|r_{k+1}-s_{k+1}\right|^{q} \leq 0
$$

which implies that

$$
\left|\frac{\partial}{\partial x} l\left(r_{k+1}, \bar{u}_{k}\right)\right| \leq \gamma
$$

and hence $r_{k+1} \in \mathcal{L}^{\gamma}$. Thus $r_{k} \in \mathcal{L}^{\gamma}$ for all $k \geq 0$. Hence, we can define $\mathcal{K}_{S}^{\bar{u}}$ as

$$
\mathcal{K}_{S}^{\bar{u}}=\bigcup_{k \geq 0} \mathcal{X}_{k}^{\bar{u}}(0)
$$

Clearly $0 \in \mathcal{K}_{S}^{\bar{u}}$. Furthermore, via time invariance and the static nature of the state feedback policy, it follows that if $x_{0} \in \mathcal{K}_{S}^{\bar{u}}$, then $x_{k} \in \mathcal{K}_{S}^{\bar{u}}, k \geq 0$. For part ii), we have already established that $x_{k} \rightarrow \mathcal{L}^{\gamma}$ as $k \rightarrow \infty$ in the proof of Corollary 1.

We now show that under certain assumptions, if there exists any policy $\bar{u} \in S$ such that $\mathcal{K}_{S}^{\bar{u}} \subset \mathcal{L}^{\gamma}$, then there exists a (suboptimal) solution to the dynamic programming equation (11) for all $x \in \mathcal{X}_{k}^{\bar{u}}, k \geq 0, x_{0} \in X_{0}$.

Theorem 8 (Necessity): In addition to A1)-A6), assume that

1) $\mathcal{F}(x, u)$ is upper semicontinuous for all $x \in \boldsymbol{R}^{n}$ and $u \in \boldsymbol{U}$

2) $l$ is independent of $u$ and satisfies the following: Given any $\gamma>0, \forall \delta>0, \exists \epsilon>0$, such that

$$
\mathcal{B}_{\epsilon}\left(\mathcal{L}^{\gamma}\right) \subset \mathcal{L}^{\gamma+\delta}
$$

Now suppose for a given $\gamma=\bar{\gamma}$, there exists a control policy $\bar{u}$ with a controlled-invariant set $\mathcal{K}_{S}^{\bar{u}}$ with $0 \in \mathcal{K}_{S}^{\bar{u}} \subset \mathcal{L}^{\bar{\gamma}}$. Furthermore, suppose that

$$
\bigcup_{x_{0} \in X_{0}} \limsup _{k \rightarrow \infty} \mathcal{X}_{k}^{\bar{u}}\left(x_{0}\right) \subset \mathcal{L}^{\bar{\gamma}} .
$$

Then for any $\delta>0$, there exists a solution $V$ to the dynamic programming equation (11), with $V(x) \geq 0, V(0)=0, \forall x \in$ $\mathcal{X}_{k}^{\bar{u}}\left(x_{0}\right), k \geq 0, x_{0} \in X_{0}$, with $\gamma=\bar{\gamma}+\delta$.
Proof: We need only establish that under $\bar{u}, \Sigma^{\bar{u}}$ is finite gain. Pick any $\delta>0$ and any $x_{0} \in X_{0}$. Now let $\epsilon>0$ be defined as in (12). Then since

$$
\limsup _{k \rightarrow \infty} \mathcal{X}_{k}^{\bar{u}}\left(x_{0}\right) \subset \mathcal{L}^{\bar{\gamma}}
$$

we have that there exists $K\left(x_{0}\right)$ such that

$$
\mathcal{X}_{k}^{\bar{u}}\left(x_{0}\right) \subset \mathcal{L}^{\bar{\gamma}+\delta}, \quad \forall k>K\left(x_{0}\right) .
$$

Also, since $\mathcal{F}(x, u)$ is upper semicontinuous, an application of Berge's theorem [25] establishes that

$$
\mathcal{X}_{k}^{\bar{u}}\left(x_{0}\right) \subset \mathcal{M}_{k}\left(x_{0}\right), \quad k=1, \cdots, K\left(x_{0}\right)
$$

where each $\mathcal{M}_{k}\left(x_{0}\right), k=1, \cdots, K\left(x_{0}\right)$ is compact. Hence, there exists a $\bar{M}\left(x_{0}\right)$, such that

$$
\begin{gathered}
J_{k}^{\bar{u}}\left(x_{0}\right)=\sup _{r, s \in \Gamma_{0, k}^{\bar{u}}\left(x_{0}\right)} \sum_{i=0}^{k-1}\left\{\left|l\left(r_{i+1}\right)-l\left(s_{i+1}\right)\right|^{q}-(\bar{\gamma}+\delta)^{q} \mid r_{i+1}\right. \\
\left.-\left.s_{i+1}\right|^{q}\right\} \leq \bar{M}\left(x_{0}\right)
\end{gathered}
$$

for all $k \geq 0$, with $J_{k}^{\bar{u}}\left(x_{0}\right) \geq 0$. Since $J_{k+1}^{\bar{u}}\left(x_{0}\right) \geq$ $J_{k}^{\bar{u}}\left(x_{0}\right), J^{\bar{u}}\left(x_{0}\right)=\lim _{k \rightarrow \infty} J_{k}^{\bar{u}}\left(x_{0}\right)$ exists and is finite. Also, if $x_{0}=0$, then $\mathcal{X}_{k}^{\bar{u}}(0) \subset \mathcal{L}^{\bar{\gamma}+\delta}$ for all $k \geq 0$, and hence $J^{\bar{u}}(0)=0$. Thus setting $\beta^{\bar{u}}\left(x_{0}\right)=J^{\bar{u}}\left(x_{0}\right)$ we conclude that $\Sigma^{\bar{u}}$ is finite gain with $\gamma=\bar{\gamma}+\delta$.

The following corollary is an immediate consequence of the theorems above.

Corollary 2: Let all the assumptions in Theorem 8 hold, however, with $l$ not necessarily independent of $u$. Let $h(x, u)=(\partial / \partial x) l(x, u)$, and assume that $\mathcal{K}_{S}^{\bar{u}}$ is such that if $x_{0} \in \mathcal{K}_{S}^{\bar{u}}$, then for any $x \in \Gamma^{\bar{u}}\left(x_{0}\right),\left|h\left(x_{k+1}, \bar{u}_{k}\right)\right| \leq \bar{\gamma}$, for all $k \geq 0$, and if $x_{0} \notin \mathcal{K}_{S}^{\bar{u}}$, then for any $\bar{x} \in$ $\Gamma^{\bar{u}}\left(x_{0}\right), \limsup _{k \rightarrow \infty}\left|h\left(x_{k+1}, \bar{u}_{k}\right)\right| \leq \bar{\gamma}$. Then for any $\delta>0$, there exists a function $V(x)$ such that $V(x) \geq 0, V(0)=0$, and $V$ satisfies (11) with $\gamma=\bar{\gamma}+\delta$ for all $x \in \mathcal{X}_{k}^{\bar{u}}\left(x_{0}\right), k \geq$ $0, x_{0} \in X_{0}$. Conversely, assume that conditions for Theorem 6 are satisfied, with $\bar{u}$ the resulting policy. Then there exists a $\mathcal{K}_{S}^{\bar{u}} \subset \mathcal{L}^{\gamma}$ with $0 \in \mathcal{K}_{S}^{\bar{u}}$ and such that: 1) for all $x_{0} \in \mathcal{K}_{S}^{\bar{u}}$ and all $x \in \Gamma^{\bar{u}}\left(x_{0}\right),\left|h\left(x_{k+1}, \bar{u}_{k}\right)\right| \leq \gamma$ and 2) for all $x_{0} \notin \mathcal{K}_{S}^{\bar{u}}$, for all $x \in \Gamma^{\bar{u}}\left(x_{0}\right), \limsup \sup _{k \rightarrow \infty}\left|h\left(x_{k+1}, \bar{u}_{k}\right)\right| \leq \gamma$.

\section{OUtPut FeEDBACK CASE}

We now consider the output feedback robust control problem. We denote the set of control policies as $O$. Hence, if $u \in O$, then $u_{k}=f\left(y_{1, k}, u_{0, k-1}\right)$.

\section{A. Finite Time}

For the finite-time case, we are only interested in the satisfaction of Condition C3) of Section II. Hence, the problem is, given $\gamma>0$ and a finite-time interval $[0, K]$, find a control policy $u \in O_{0, K-1}$ such that there exists a finite quantity $\beta_{K}^{u}(x)$ with $\beta_{K}^{u}(0)=0$ and

$$
\begin{aligned}
& \sum_{i=0}^{K-1}\left|l\left(r_{i+1}, u_{i}\right)-l\left(s_{i+1}, u_{i}\right)\right|^{q}-\gamma^{q}\left|r_{i+1}-s_{i+1}\right|^{q} \\
& \leq \beta_{K}^{u}\left(x_{0}\right), \quad \forall r, s \in \Gamma_{0, K}^{u}\left(x_{0}\right), \quad \forall x_{0} \in X_{0} .
\end{aligned}
$$


1) Dynamic Game: In this subsection, we transform the output feedback robust control problem to a dynamic game. We introduce the function space

$$
\mathcal{E}=\left\{p: \boldsymbol{R}^{n} \rightarrow \boldsymbol{R}^{*}\right\}
$$

For $u \in O_{0, K-1}$ and $p \in \mathcal{E}$ define a functional $J_{p, k}(u)$ by

$$
\begin{aligned}
J_{p, k}(u) \triangleq \sup _{x_{0} \in X_{0}} \sup _{r, s \in \Gamma^{u}\left(x_{0}\right)} & \left\{p\left(x_{0}\right)+\sum_{i=1}^{k} \mid l\left(s_{i}, u_{i-1}\right)\right. \\
& \left.-\left.l\left(r_{i}, u_{i-1}\right)\right|^{q}-\gamma^{q}\left|s_{i}-r_{i}\right|^{q}\right\}
\end{aligned}
$$

for $k=0, \cdots, K$.

As will be shown, we follow the convention that the supremum over an empty set is $-\infty$. The finite-gain property of $\Sigma^{u}$ can now be expressed in terms of $J$ as follows.

Lemma 2: $\Sigma^{u}$ is finite gain on $[0, K]$, if and only if there exists a finite $\beta_{K}^{u} \geq 0$ on $X_{0}$, with $\beta_{K}^{u}(0)=0$, such that

$$
J_{-\beta_{K}^{u}, k}(u) \leq 0, \quad k=0, \cdots, K .
$$

For notational convenience, we introduce the following pairing for any two functions $a, b \in \mathcal{E}$ :

$$
(a, b)=\sup _{x \in \boldsymbol{R}^{n}}\{a(x)+b(x)\} .
$$

Lemma 3: If $\Sigma^{u}$ is finite gain on $[0, K]$, then

$$
(p, 0) \leq J_{p, k}(u) \leq\left(p, \beta_{K}^{u}\right), \quad k=1, \cdots, K .
$$

Proof: Set $r=s \in \Gamma^{u}\left(x_{0}\right)$ in (13). Then clearly

$$
(p, 0) \leq J_{p, k}(u), \quad k=1, \cdots, K .
$$

Since $\Sigma^{u}$ is finite gain on $[0, K]$, this implies that for any $r, s \in \Gamma_{0, k}^{u}\left(x_{0}\right), x_{0} \in X_{0}$, and $k \in[1, K]$

$$
\begin{aligned}
p\left(x_{0}\right) & +\sum_{i=1}^{k}\left|l\left(s_{i}, u_{i-1}\right)-l\left(r_{i}, u_{i-1}\right)\right|^{q}-\gamma^{q}\left|s_{i}-r_{i}\right|^{q} \\
& \leq p\left(x_{0}\right)+\beta_{K}^{u}\left(x_{0}\right) \leq\left(p, \beta_{K}^{u}\right) .
\end{aligned}
$$

Hence, $J_{p, k}(u) \leq\left(p, \beta_{K}^{u}\right)$.

Thus, solving the finite-time problem amounts to finding a control policy $u \in O_{0, K-1}$ which minimizes $J_{p, K}(u)$. This also motivates characterizing values of $p \in \mathcal{E}$ that yield bounded values for $J_{p, K}(u)$. Thus, given that $\Sigma^{u}$ is finite gain on $[0, K]$, we define

$$
\operatorname{dom} J_{, K}(u)=\left\{p \in \mathcal{E}:(p, 0),\left(p, \beta_{K}^{u}\right) \text { is finite }\right\} .
$$

2) Information State Formulation: Motivated by [14] and [15], for a fixed $y_{1, k} \in \Delta_{1, k}^{u}\left(X_{0}\right)$ and $u_{1, k-1}$, we define the information state $p_{k} \in \mathcal{E}$ by

$p_{k}(x)$

$$
\begin{array}{r}
\triangleq \sup _{x_{0} \in X_{0}} \sup _{r, s \in \Gamma_{0, k}^{u, y}\left(x_{0}\right)}\left\{p_{0}\left(x_{0}\right)+\sum_{i=1}^{k}\left|l\left(s_{i}, u_{i-1}\right)-l\left(r_{i}, u_{i-1}\right)\right|^{q}\right. \\
\left.-\gamma^{q}\left|r_{i}-s_{i}\right|^{q} \mid r_{k}=x\right\} .
\end{array}
$$

Remark 7: The functional $p_{0} \in \mathcal{E}$ in (14) is supposed to reflect any a priori knowledge concerning the initial state $x_{0}$. In particular, one has $p_{0}(x)=-\infty$, for all $x_{0} \notin X_{0}$. Furthermore, if one knew the initial state value with certainty, i.e., $x_{0}=\bar{x}$, one could then set $p_{0}=\delta_{\{\bar{x}\}}$.

Clearly, if $\Sigma^{u}$ is finite gain, then

$$
-\infty \leq p_{k}(x) \leq\left(p_{0}, \beta_{K}^{u}\right)<+\infty
$$

and a finite lower bound for $p_{k}(x)$ is obtained for all feasible $x \in \boldsymbol{R}^{n}$.

Now, define $H(p, u, y) \in \mathcal{E}$ by

$$
H(p, u, y)(x) \triangleq \sup _{\xi \in \boldsymbol{R}^{n}}\{p(\xi)+B(\xi, x, u, y)\}
$$

where the function $B$ is defined by the equation shown at the bottom of the page.

Lemma 4: The information state is the solution of the following recursion:

$$
\begin{aligned}
p_{k+1} & =H\left(p_{k}, u_{k}, y_{k+1}\right), \quad k=0, \cdots, K-1 \\
p_{0} & \in \mathcal{E}
\end{aligned}
$$

with $p_{0}(x)=-\infty$, for all $x \notin X_{0}$.

Proof: We use induction. Assume that (16) is true for $0, \cdots, k$; we must show that $p_{k+1}$ equals $H\left(p_{k}, u_{k}, y_{k+1}\right)$. Now

$$
\begin{aligned}
H\left(p_{k}, u_{k}, y_{k+1}\right)(x) & \\
= & \sup _{\xi \in \boldsymbol{R}^{n}}\left\{p_{k}(\xi)+B\left(\xi, x, u_{k}, y_{k+1}\right)\right\} \\
= & \sup _{\xi \in \boldsymbol{R}^{n}}\left\{p_{k}(\xi)\right. \\
& +\sup _{s \in \mathcal{F}\left(\xi, u_{k}\right)}\left(\left|l\left(x, u_{k}\right)-l\left(s, u_{k}\right)\right|^{q}\right. \\
& \left.-\gamma^{q}|x-s|^{q}\right) \mid y_{k+1} \in \mathcal{G}\left(\xi, u_{k}\right) \\
& \left.x \in \mathcal{F}\left(\xi, u_{k}\right)\right\} \\
= & p_{k+1}(x)
\end{aligned}
$$

by the definition (14) for $p_{k}$ and $p_{k+1}$.

$$
B(\xi, x, v, y) \triangleq \begin{cases}\sup _{s \in \mathcal{F}(\xi, v)}\left\{|l(x, v)-l(s, v)|^{q}-\gamma^{q}|x-s|^{q}\right\}, & \text { if }\left\{\begin{array}{l}
x \in \mathcal{F}(\xi, v) \\
y \in \mathcal{G}(\xi, v)
\end{array}\right. \\
-\infty, & \text { else. }\end{cases}
$$


Remark 8: The relationship between the information state and the indicator function of the feasible sets was established in [15]. In particular, it was established that if $p_{0}=\delta_{\left\{x_{0}\right\}}$, then $p_{k}(x) \geq 0$ if and only if $x \in \mathcal{X}_{k}^{y, u}\left(x_{0}\right)$, where $\mathcal{X}_{k}^{y, u}\left(x_{0}\right)$ is the set of feasible states at time $k$, given $u_{0, k-1}$ and $y_{1, k}$ (i.e., the set of state values the system could achieve at time $k$, given the observation sequences $u_{0, k-1}, y_{1, k}$ ).

Theorem 9: For $u \in O_{0, K-1}, p \in \mathcal{E}$, such that $J_{p, K}(u)$ is finite, we have

$$
J_{p, k}(u)=\sup _{y_{1, k} \in \Delta_{1, k}^{u}\left(X_{0}\right)}\left\{\left(p_{k}, 0\right) \mid p_{0}=p\right\}, \quad k \in[0, K]
$$

where $p_{k}$ is generated via (16).

Proof: We have the equation shown at the bottom of the page.

Remark 9: This representation theorem is actually a separation principle and enables us to express the finite-gain property of $\Sigma^{u}$ in terms of the information state $p$.

Corollary 3: For any output feedback controller $u \in$ $O_{0, K-1}$, the closed-loop system $\Sigma^{u}$ is finite gain on $[0, K]$ if and only if the information state $p_{k}$ satisfies

$$
\sup _{y_{1, k} \in \Delta_{1, k}^{u}\left(X_{0}\right)}\left\{\left(p_{k}, 0\right) \mid p_{0}=-\beta_{K}^{u}\right\} \leq 0, \quad \forall k \in[0, K]
$$

for some finite $\beta_{K}^{u}\left(x_{0}\right) \geq 0, x_{0} \in X_{0}$, with $\beta_{K}^{u}(0)=0$.

Remark 10: Thus the name information state for $p$ is justified, since $p_{k}$ contains all the information relevant to the finite-gain property of $\Sigma^{u}$ that is available in the observations $y_{1, k}$.

The information state dynamics (16) may be regarded as a new (infinite dimensional) control system $\Xi$, with control $u$ and uncertainty parameterized by $y$. The state $p_{k}$ and the disturbance $y_{k}$ are available to the controller, so the original output feedback dynamic game is equivalent to a new game with full information. The cost is now given by (17). Note that now the control will depend only on the information state. Hence, the controller has a separated structure.

We now need an appropriate class $I_{i, K-1}$ of controllers, which feedback this new state variable. A control $u$ belongs to $I_{i, K-1}$, if for each $k \in[i, K-1]$, there exists a map $\bar{u}_{k}$ from a subset of $\mathcal{E}^{k-i+1}$ (sequences $p_{i, k}$ ) into $\boldsymbol{U}$, such that $u_{k}=\bar{u}\left(p_{i, k}\right)$. Note that since $p_{k}$ depends on the observable information $y_{i, k}, I_{0, k-1} \subset O_{0, k-1}$, for $k=1, \cdots, K$.

3) Solution to the Finite-Time Output Feedback Robust Control Problem: We use dynamic programming to solve the game. Define the value function by

$$
M_{k}(p)=\inf _{u \in O_{0, k-1}} \sup _{y \in \Delta_{1, k}^{u}\left(X_{0}\right)}\left\{\left(p_{k}, 0\right) \mid p_{0}=p\right\}
$$

for $k \in[0, K]$, and the corresponding dynamic programming equation is

$$
M_{k}(p)=\inf _{u \in \boldsymbol{U}^{t}} \sup _{y \in \boldsymbol{R}^{t}}\left\{M_{k-1}(H(p, u, y))\right\}, \quad k \in[1, K]
$$

with the initial condition

$$
M_{0}(p)=(p, 0)
$$

Remark 11: In the above equations, we have inverted the time index to enable ease of exposition when dealing with the infinite-time case. Since the system is assumed to be time invariant, it does not matter if we write the equations as above or as

$\tilde{M}_{k}(p)=\inf _{u \in \boldsymbol{U}} \sup _{y \in \boldsymbol{R}^{t}}\left\{\tilde{M}_{k+1}(H(p, u, y))\right\}, \quad k \in[0, K-1]$ with the initial condition

$$
\tilde{M}_{K}(p)=(p, 0)
$$

as long as we invert the index of the control policy obtained by solving (19).

Define for a function $M: \mathcal{E} \rightarrow \boldsymbol{R}^{*}$

$$
\operatorname{dom} M=\{p \in \mathcal{E} \mid M(p) \text { finite }\} \text {. }
$$

Theorem 10 (Necessity): Assume that $\bar{u} \in O_{0, K-1}$ solves the finite-time output feedback robust control problem. Then, there exists a solution $M$ to the dynamic programming equation (19) such that $\operatorname{dom} J_{, K}(\bar{u}) \subset \operatorname{dom} M_{k}, M_{k}\left(-\beta_{K}^{\bar{u}}\right)=$ $0, M_{k}(p) \geq(p, 0), p \in \operatorname{dom} M_{k}, k \in[0, K]$.

Proof: For $p \in \operatorname{dom} J_{\cdot, K}(\bar{u})$, define $M_{k}(p)$ by (18).

Then

$$
M_{k}(p)=\inf _{u \in O_{0, k-1}} J_{p, k}(u) .
$$

Now, we also have

$$
\begin{aligned}
M_{k}(p)= & \inf _{u \in O_{0, k-1}} \sup _{x_{0} \in X_{0}} \sup _{r, s \in \Gamma_{0, k}^{u}\left(x_{0}\right)}\left\{p\left(x_{0}\right)\right. \\
& \left.+\sum_{i=1}^{k}\left|l\left(s_{i}, u_{i-1}\right)-l\left(r_{i}, u_{i-1}\right)\right|^{q}-\gamma^{q}\left|s_{i}-r_{i}\right|^{q}\right\} .
\end{aligned}
$$

For $u=\bar{u}$, by using the finite-gain property for $\Sigma^{\bar{u}}$ we get

$$
\begin{aligned}
& M_{k}(p) \\
& \leq \sup _{x_{0} \in X_{0}} \sup _{r, s \in \Gamma_{0, k}^{\bar{u}}\left(x_{0}\right)}\left\{p\left(x_{0}\right)+\sum_{i=1}^{k}\left|l\left(s_{i}, \bar{u}_{i-1}\right)-l\left(r_{i}, \bar{u}_{i-1}\right)\right|^{q}\right. \\
& \left.\quad-\gamma^{q}\left|s_{i}-r_{i}\right|^{q}\right\} \\
& \leq\left(p, \beta_{K}^{\bar{u}}\right) .
\end{aligned}
$$

$$
\begin{aligned}
\sup _{y_{1, k} \in \Delta_{1, k}^{u}\left(X_{0}\right)}\left\{\left(p_{k}, 0\right) \mid p_{0}=p\right\} & =\sup _{x_{0} \in X_{0}} \sup _{y_{1, k} \in \Delta_{1, k}^{u}\left(x_{0}\right)} \sup _{r, s \in \Gamma_{0, k}^{u, y}\left(x_{0}\right)}\left\{p\left(x_{0}\right)+\left.\sum_{i=1}^{k} l\left(s_{i}, u_{i-1}\right)\right|^{q}-\gamma^{q}\left|r_{i}-s_{i}\right|^{q}\right\} \\
& =\sup _{x_{0} \in X_{0}} \sup _{r, s \in \Gamma_{0, k}^{u}\left(x_{0}\right)}\left\{p\left(x_{0}\right)+\sum_{i=1}^{k}\left|l\left(s_{i}, u_{i-1}\right)-l\left(r_{i}, u_{i-1}\right)\right|^{q}-\gamma^{q}\left|r_{i}-s_{i}\right|^{q}\right\} \\
& =J_{p, k}(u)
\end{aligned}
$$


Thus, dom $J_{, K}(\bar{u}) \subset \operatorname{dom} M_{k}$. Also

$$
M_{k} \cdot(p) \geq(p, 0) .
$$

Also $-\beta_{K}^{\bar{u}} \leq 0$, with $\beta_{K}^{\bar{u}}(0)=0$, and hence $M_{k}\left(-\beta_{K}^{\bar{u}}\right)=0$.

Theorem 11 (Sufficiency): Assume there exists a solution $M$ to the dynamic programming equation (19) on some nonempty domain dom $M_{k}$, such that $-\beta \in$ dom $M_{k}\left(\beta(x) \geq 0\right.$ and finite for all $x \in X_{0}$, with $\beta(0)=0), M_{k}(-\beta)=0, M_{k}(p) \geq(p, 0), k \in[0, K]$. Let $u^{*} \in I_{0, K-1}$ be a policy such that $u_{k}^{*}=\bar{u}_{K-k}^{*}\left(p_{k}\right), k=$ $0, \cdots, K-1$, where $\bar{u}_{k}^{*}(p)$ achieves the minimum in (19). Let $p_{0}=-\beta$ and $p_{k}$ be the corresponding information state trajectory with $p_{k} \in \operatorname{dom} M_{K-k}, k=1, \cdots, K$. Then $u^{*}$ solves the finite-time output feedback robust control problem.

Proof: We see that

$$
M_{K}(p)=J_{p, K}\left(u^{*}\right) \leq J_{p, K}(u)
$$

for all $u \in O_{0, K-1}, p \in \operatorname{dom} M_{K}$. Now

$$
\sup _{y \in \Delta_{1, K}^{u^{* *}}\left(X_{0}\right)}\left\{\left(p_{K}, 0\right) \mid p_{0}=-\beta\right\} \leq M_{K}(-\beta)=0
$$

which implies by Corollary 3 that $\Sigma^{u^{*}}$ is finite gain, and hence $u^{*}$ solves the finite-time output feedback robust control problem.

Corollary 4: If the finite-time output feedback robust control problem is solvable by an output feedback controller $\bar{u} \in O_{0, K-1}$, then it is also solvable by an information state feedback controller $u^{*} \in I_{0, K-1}$.

\section{B. Infinite-Time Case}

For the infinite-time case, we need to satisfy the conditions C1)-C3) stated in Section II. We pass to the limit as $K \rightarrow \infty$ in the dynamic programming equation (19)

$$
\lim _{k \rightarrow \infty} M_{k}(p)=M(p)
$$

where $M_{k}(p)$ is defined by (18), to obtain a stationary version of (19)

$$
M(p)=\inf _{u \in \boldsymbol{U}} \sup _{y \in \boldsymbol{R}^{t}}\{M(H(p, u, y))\} .
$$

1) Dissipation Inequality: The following lemma is a consequence of Corollary 3.

Lemma 5: For any $u \in O$, the closed-loop system $\Sigma^{u}$ is finite gain if and only if the information state satisfies

$$
\sup _{k \geq 1} \sup _{y \subset \Delta_{1, k}^{u}\left(X_{0}\right)}\left\{\left(p_{k}, 0\right) \mid p_{0}=-\beta^{u}\right\} \leq 0
$$

for some $\beta^{u}\left(x_{0}\right) \geq 0$, finite on $X_{0}$, with $\beta^{u}(0)=0$.

By using Lemma 5 we say that the information state system $\Xi^{u}[(16)$ with information state feedback $u \in I]$ is finite gain if and only if the information state $p_{k}$ satisfies (21) for some finite $\beta^{u}\left(x_{0}\right)$, with $\beta^{u}(0)=0$. If $\Sigma^{u}$ is finite gain, we write

$$
\operatorname{dom} J .(u)=\left\{p \in \mathcal{E} \mid(p, 0),\left(p, \beta^{u}\right) \text { finite }\right\}
$$

where for every $p \in \mathcal{E}$ we define $J_{p}(u)$ as $J_{p}(u)=\sup _{k \geq 0}$ $J_{p, k}(u)$.
We say that the information state system $\Xi^{\bar{u}}$ is finite-gain dissipative if there exists a function (storage function) $M(p)$, such that dom $M$ contains $-\beta(\beta \geq 0$ and finite for all $x \in X_{0}$, with $\left.\beta(0)=0\right), M(p) \geq(p, 0), M(-\beta)=0$, and satisfies the following dissipation inequality:

$$
M(p) \geq \sup _{y \in \boldsymbol{R}^{t}}\{M(H(p, \bar{u}(p), y))\}, \quad \forall p \in \operatorname{dom} M .
$$

Note that if $\Xi^{\bar{u}}$ is finite-gain dissipative, and $p \in \operatorname{dom} M$, then $H(p, \bar{u}(p), y) \in \operatorname{dom} M$ for all $y \in \boldsymbol{R}^{t}$. Consequently, $p_{0} \in \operatorname{dom} M$, implies $p_{k} \in \operatorname{dom} M, \forall k>0$.

Lemma 6: $M_{k}$ is monotone nondecreasing, i.e.,

$$
M_{k-1}(p) \leq M_{k}(p) .
$$

Proof: Note that

$$
\begin{aligned}
& M_{k}(p) \\
& =\sup _{x_{0} \in X_{0}} \sup _{r, s \in \Gamma_{0, k}^{u}\left(x_{0}\right)}\left\{p\left(x_{0}\right)+\sum_{i=1}^{k}\left|l\left(r_{i}, u_{i-1}\right)-l\left(s_{i}, u_{i-1}\right)\right|^{q}\right. \\
& \left.-\gamma^{q}\left|s_{i}-r_{i}\right|^{q}\right\} .
\end{aligned}
$$

Then for any $\epsilon>0$, choose $x_{0}^{\prime} \in X_{0}$, and $r^{\prime}, s^{\prime} \in \Gamma_{0, k-1}^{u}\left(x_{0}^{\prime}\right)$ such that

$$
\begin{aligned}
M_{k-1}(p) \leq & p\left(x_{0}^{\prime}\right)+\sum_{i=1}^{k-1}\left|l\left(r_{i}^{\prime}, u_{i-1}\right)-l\left(s_{i}^{\prime}, u_{i-1}\right)\right|^{q} \\
& -\gamma^{q}\left|r_{i}^{\prime}-s_{i}^{\prime}\right|^{q}+\epsilon .
\end{aligned}
$$

Let $x_{0}=x_{0}^{\prime}$, and define $r, s \in \Gamma_{0, k}^{u}\left(x_{0}\right)$ by $r=r^{\prime}, s=s^{\prime}$ on $[0, k-1]$, and $r_{k}=s_{k}$. Then

$$
\begin{aligned}
M_{k}(p) \geq & p\left(x_{0}\right)+\sum_{i=1}^{k}\left|l\left(r_{i}, u_{i-1}\right)-l\left(s_{i}, u_{i-1}\right)\right|^{q} \\
& -\gamma^{q}\left|r_{i}-s_{i}\right|^{q} \\
& \geq p\left(x_{0}^{\prime}\right)+\sum_{i=1}^{k-1}\left|l\left(r_{i}^{\prime}, u_{i-1}\right)-l\left(s_{i}^{\prime}, u_{i-1}\right)\right|^{q} \\
& -\gamma^{q}\left|r_{i}^{\prime}-s_{i}^{\prime}\right|^{q}+\left|l\left(r_{k}, u_{k-1}\right)-l\left(s_{k}, u_{k-1}\right)\right|^{q} \\
& \geq M_{k-1}(p)-\epsilon .
\end{aligned}
$$

Since $\epsilon>0$ is arbitrary, letting $\epsilon \rightarrow 0+$ gives

$$
M_{k}(p) \geq M_{k-1}(p) \text {. }
$$

We are now in a position to prove a version of the bounded real lemma for the information state system $\Xi$.

Theorem 12: Let $u \in I$. Then the information state system $\Xi^{u}$ is finite gain if and only if it is finite-gain dissipative.

Proof $i$ ): Assume that $\Xi^{u}$ is finite-gain dissipative. Then by the dissipation inequality (22)

$$
M\left(p_{k}\right) \leq M\left(p_{0}\right), \quad \forall k>0, \quad \forall y \in \Delta_{1, k}^{u}\left(X_{0}\right) .
$$

Setting $p_{0}=-\beta$, and using the fact that $M(p) \geq(p, 0)$, we get

$$
\left(p_{k}, 0\right) \leq M(-\beta)=0, \quad \forall k>0, \quad \forall y \in \Delta_{1, k}^{u}\left(x_{0}\right) .
$$

Therefore $\Xi^{u}$ is finite gain, with $p_{0}=-\beta$. 
ii): Assume $\Xi^{u}$ is finite gain. Then

$$
(p, 0) \leq J_{p, k}(u) \leq\left(p, \beta^{u}\right), \quad \forall k \geq 0, p \in \operatorname{dom} J .(u) .
$$

Writing $M_{k}(p)=J_{p, k}(u)$ so that

$$
(p, 0) \leq M_{k}(p) \leq\left(p, \beta^{u}\right), \quad k \geq 0, p \in \operatorname{dom} J .(u) .
$$

By Lemma $6, M_{k}$ is monotone nondecreasing. Therefore

$$
M_{a}(p)=\lim _{k \rightarrow \infty} M_{k}(p)
$$

exists and is finite on dom $M_{a}$, which contains dom $J .(u)$.

To show that $M_{a}$ satisfies (22), fix $p \in \operatorname{dom} M_{a}, y \in \boldsymbol{R}^{t}$ and $\epsilon>0$. Select $k>0$ and $\tilde{y}_{1, k-1}$ such that

$$
M_{a}(H(p, u(p), y)) \leq\left(\tilde{p}_{k-1}, 0\right)+\epsilon
$$

where $\tilde{p}_{j}, j=0, \cdots, k-1$ is the information state trajectory generated by $\tilde{y}$, with $\tilde{p}_{0}=H(p, u(p), y)$.

Define

$$
y_{i}= \begin{cases}y, & \text { if } i=1 \\ \tilde{y}_{i-1}, & \text { if } i=2, \cdots, k\end{cases}
$$

and let $p_{j}, j=0, \cdots, k$ denote the corresponding information state trajectory with $p_{0}=p$. Then

$$
\begin{aligned}
M_{a}(p) & \geq\left(p_{k}, 0\right) \\
& =\left(\tilde{p}_{k-1}, 0\right) \\
& \geq M_{a}(H(p, u(p), y))-\epsilon .
\end{aligned}
$$

Since $y$ and $\epsilon$ are arbitrary, we have

$$
M_{a}(p) \geq \sup _{y \in \boldsymbol{R}^{t}} M_{a}(H(p, u(p), y))
$$

Hence, $M_{a}$ solves the dissipation inequality. Also, by definition $(p, 0) \leq M_{a}(p) \leq\left(p, \beta^{u}\right)$. This implies that $M_{a}\left(-\beta^{u}\right)=$ 0 . Thus, $\Xi^{u}$ is finite-gain dissipative.

We now again assume that $\Sigma^{u}$ satisfies A7).

Theorem 13: Let $u \in I$. If $\Xi^{u}$ is finite-gain dissipative and $\Sigma^{u}$ satisfies assumption A7), then $\Sigma^{u}$ is weakly asymptotically stable.

Proof: Inequality (22) implies

$$
\begin{aligned}
\sup _{x_{0} \in X_{0}} \sup _{r, s \in \Gamma_{0, k}^{u}\left(x_{0}\right)}\{ & \left\{p\left(x_{0}\right)+\sum_{i=1}^{k} \mid l\left(r_{i}, u_{i-1}\right)\right. \\
& \left.-\left.l\left(s_{i}, u_{i-1}\right)\right|^{q}-\gamma^{q}\left|s_{i}-r_{i}\right|^{q}\right\} \leq M(p)
\end{aligned}
$$

for all $k \geq 1$. Let $x_{0} \in X_{0}$, and let $p=-\beta^{u}$. Then the above gives

$$
\begin{aligned}
\sup _{r, s \in \Gamma_{0, k}^{u}\left(x_{0}\right)} & \left\{\sum_{i=1}^{k}\left|l\left(r_{i}, u_{i-1}\right)-l\left(s_{i}, u_{i-1}\right)\right|^{q}\right. \\
& \left.-\gamma^{q}\left|s_{i}-r_{i}\right|^{q}\right\} \leq M(p)+\beta^{u}\left(x_{0}\right)<+\infty .
\end{aligned}
$$

For any $\bar{r} \in \Gamma_{0, k}^{u}\left(x_{0}\right)$, there is a sequence

$$
\begin{gathered}
W_{i}^{u}=\sup _{r^{\prime} \in \mathcal{F}\left(\bar{r}_{i}, u_{i}\right)}\left\{\left|l\left(r^{\prime}, u_{i}\right)-l\left(\bar{r}_{i+1}, u_{i}\right)\right|^{q}\right. \\
\left.\quad-\gamma^{q}\left|r^{\prime}-\bar{r}_{i+1}\right|^{q}\right\} \\
i=0, \cdots, k-1 \\
\geq 0 .
\end{gathered}
$$

Also, from above we obtain that

$$
\sum_{i=0}^{k} W_{i}^{u}<+\infty, \quad \forall k>0 .
$$

Hence, $W_{i}^{u} \rightarrow 0$, as $i \rightarrow \infty$ and by Corollary 6 and Assumption A7)

$$
0 \in \liminf _{i \rightarrow \infty} \mathcal{F}\left(\bar{r}_{i}, u_{i}\right)
$$

Hence, $\Sigma^{u}$ is weakly asymptotically stable.

Corollary 5: If $\Xi^{u}$ is finite-gain dissipative, then $\Sigma^{u}$ is ultimately bounded.

Proof: The proof is similar to that of Corollary 1.

We also need to show that the information state system $\Xi^{u}$ is stable.

Theorem 14: Let $u \in I$. If $\Xi^{u}$ is finite-gain dissipative, then $\Xi^{u}$ is stable on all feasible $x \in \boldsymbol{R}^{n}$.

Proof: Inequality (22) implies that

$$
p_{k}(x) \leq\left(p_{k}, 0\right) \leq M\left(p_{0}\right)<+\infty
$$

for all $p_{0} \in \operatorname{dom} M$ and for all $k \geq 0$. For the lower bound, note that by definition (14)

$$
\begin{aligned}
p_{k}(x)=\sup _{x_{0} \in X_{0}} \sup _{r, s \in \Gamma_{0, k}^{u}\left(x_{0}\right)}\{ & p_{0}\left(x_{0}\right)+\sum_{i=1}^{k} \mid l\left(s_{i}, u_{i-1}\right) \\
& -\left.l\left(r_{i}, u_{i-1}\right)\right|^{q} \\
& \left.-\gamma^{q}\left|r_{i}-s_{i}\right|^{q} \mid r_{k}=x\right\} .
\end{aligned}
$$

For any $x_{0} \in X_{0}$, this implies that for any feasible $x \in \boldsymbol{R}^{n}$

$$
p_{k}(x) \geq p_{0}\left(x_{0}\right)>-\infty, \quad \forall k \geq 0 .
$$

Therefore, $\Xi^{u}$ is stable.

2) Solution to the Output Feedback Robust Control Problem: As in the state feedback case, it can be inferred from the previous results that the controlled dissipation inequality (22) is both a necessary and sufficient condition for the solvability of the output feedback robust control problem.

However, we now state necessary and sufficient conditions for the solvability of the output feedback robust control problem in terms of dynamic programming equalities.

Theorem 15 (Necessity): Assume that there exists a controller $\bar{u} \in O$ which solves the output feedback robust control problem. Then there exists a function $M(p)$ such that $\operatorname{dom} J \cdot(\bar{u}) \subset \operatorname{dom} M(p), M(p) \geq(p, 0), M\left(-\beta^{\bar{u}}\right)=0$ and $M$ solves the stationary dynamic programming equation

$$
M(p)=\inf _{u \in \boldsymbol{U}} \sup _{y \in \boldsymbol{R}^{t}}\{M(H(p, u, y))\}
$$

for all $p \in \operatorname{dom} J \cdot(\bar{u})$. 

follows:

Proof: For $p \in \operatorname{dom} J .(\bar{u})$, define $M_{k}(p), k=0, \cdots$ as

$$
\begin{aligned}
& M_{k}(p)=\inf _{u \in \boldsymbol{U}} \sup _{y \in \boldsymbol{R}^{t}} M_{k-1}(H(p, u, y)) \\
& M_{0}(p)=(p, 0)
\end{aligned}
$$

Clearly

$$
(p, 0) \leq M_{k}(p) \leq\left(p, \beta^{\bar{u}}\right)<+\infty, \quad \forall p \in \operatorname{dom} J \cdot(\bar{u}) .
$$

Furthermore, a modification of Lemma 6 establishes that

$$
M_{k+1}(p) \geq M_{k}(p), \quad \forall p \in \operatorname{dom} J \cdot(\bar{u}) .
$$

Hence

$$
M_{k}(p) \rightarrow M(p) \text { as } k \rightarrow \infty
$$

and $M(p)$ satisfies (23) for all $p \in \operatorname{dom} J .(\bar{u})$.

Furthermore, $\operatorname{dom} J .(\bar{u}) \subset \operatorname{dom} M(p)$ and $(p, 0) \leq M(p)$ $\leq\left(p, \beta^{\bar{u}}\right)$. Thus, since $-\beta^{\bar{u}} \leq 0, \beta^{\bar{u}}(0)=0, M\left(-\beta^{\bar{u}}\right)=0$.

Theorem 16 (Sufficiency): Assume that there exists a solution $M$ to the stationary dynamic programming equation (23) on some nonempty domain $\operatorname{dom} M$ such that $-\beta \in \operatorname{dom} M$, $\left(\beta(x) \geq 0\right.$, and finite $\forall x \in X_{0}$, with $\left.\beta(0)=0\right), M(-\beta)=0$, and $M(p) \geq(p, 0)$. Let $\bar{u} \in I$ be a policy such that $\bar{u}(p)$ achieves the minimum in (23). Let $p_{0}=-\beta$, and let $p_{k}$ be the corresponding information state trajectory satisfying $p_{k} \in$ dom $M, k=0,1, \cdots$. Then, $\bar{u} \in I$ solves the information state feedback robust control problem if the closed-loop system $\Sigma^{\bar{u}}$ satisfies Assumption A7).

Proof: Since $M$ satisfies (23), $\Sigma^{\bar{u}}$ satisfies (22) with equality. Hence, $\Xi^{\bar{u}}$ is finite-gain dissipative and by Theorem 12, $\Xi^{\bar{u}}$ is finite gain. Furthermore, Theorem 13 establishes that $\Sigma^{\bar{u}}$ is weakly asymptotically stable, and by Corollary $5 \Sigma^{\bar{u}}$ is ultimately bounded. Also by Theorem 14, $\Xi^{\bar{u}}$ is stable for all feasible $x \in \operatorname{Re}^{n}$.

3) Characterization of Controlled-Invariant Sets: For an output feedback policy $u \in O$, let $\mathcal{K}_{O}^{u}$ be defined as that set for which: 1) $0 \in \mathcal{K}_{O}^{u}$ and 2) if $x_{0}=0$, then $x_{k} \in \mathcal{K}_{O}^{u}$ for all $k \geq 0$. Hence, unlike the state feedback case, we require that $\mathcal{K}_{O}^{u}$ be controlled-invariant only for all trajectories starting from zero. This allows us to define such a set without referring to the states of the dynamic controller. The results in this subsection follow very much along the lines of the state feedback case, and we present them (omitting the proofs) for completeness.

Theorem 17 (Sufficiency): Suppose the conditions of Theorem 16 are satisfied with the corresponding policy $\bar{u} \in I$. Then: 1) there exists a $\mathcal{K}_{O}^{\bar{u}} \subset \mathcal{L}^{\gamma}$, with $0 \in \mathcal{K}^{\bar{u}}$ and 2) if $x_{0} \neq 0$, then $x_{k} \rightarrow \mathcal{L}^{\gamma}$ as $k \rightarrow \infty$.

Theorem 18 (Necessity): In addition to A1)-A6), assume that

1) $\mathcal{F}(x, u)$ is upper semicontinuous for all $x \in \boldsymbol{R}^{n}, u \in \boldsymbol{U}$;

2) $l$ is independent of $u$, and satisfies the following: Given any $\gamma>0$, for all $\delta>0$, there exists an $\epsilon>0$ such that

$$
\mathcal{L}^{\gamma}+\mathcal{B}_{\epsilon}(0) \subset \mathcal{L}^{\gamma+\delta}
$$

Now suppose for a given $\gamma=\bar{\gamma}$, there exists an output feedback policy $\bar{u} \in O$, such that

$$
\bigcup_{x_{0} \in X_{0}} \limsup _{k \rightarrow \infty} \mathcal{X}_{k}^{\bar{u}}\left(x_{0}\right) \subset \mathcal{L}^{\bar{\gamma}}
$$

and $0 \in \mathcal{K}_{O}^{\bar{u}} \subset \mathcal{L}^{\bar{\gamma}}$. Then for any $\delta>0$, and with $\gamma=$ $\bar{\gamma}+\delta$, there exists a function $M(p)$, such that dom $J \cdot(\bar{u}) \subset$ dom $M(p), M(p) \geq(p, 0), M\left(-\beta^{\bar{u}}\right)=0$ and $M$ solves the stationary dynamic programming equation

$$
M(p)=\inf _{u \in \boldsymbol{U}} \sup _{y \in \boldsymbol{R}^{t}}\{M(H(p, u, y))\} .
$$

Remark 12: The output feedback counterpart of Corollary 2 immediately follows, where one requires that

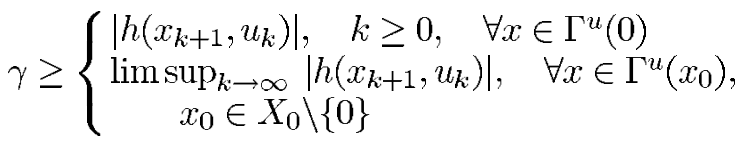

with $h$ defined as in Corollary 2 .

\section{Certainty Equivalence}

In this section, we briefly consider certainty equivalence controllers for the infinite-time case. The primary motivation for considering such controllers is the complexity associated with solving (23), which is a dynamic programming equation on what is in general an infinite-dimensional space $(\mathcal{E})$. Let $p_{k} \in \mathcal{E}$ be the information state trajectory generated by the system, let $V$ be the solution to the dynamic programming equation (11) corresponding to the state feedback robust control problem, and let $u_{F}$ be the corresponding state feedback policy. A standing assumption throughout this section is that all min, and max are achieved. Following Whittle [26] identifying $p_{k}$ as the past stress and $V$ as the future stress, compute

$$
\hat{x}_{k} \in \arg \max _{x \in \boldsymbol{R}^{n}}\left\{p_{k}(x)+V(x)\right\}
$$

and use the feedback policy $\left.u_{(} p_{k}\right)=u_{F}\left(\hat{x}_{k}\right)$. Before proceeding further, we define

$$
\begin{aligned}
Q_{U}^{p}(x, u) \triangleq & \left\{p(x)+\sup _{r, s \in \mathcal{F}(x, u)}\left\{|l(r, u)-l(s, u)|^{q}\right.\right. \\
& \left.\left.-\gamma^{q}|r-s|^{q}+U(r)\right\}\right\} .
\end{aligned}
$$

The result follows from the definition of $Q_{U}^{p}$ and the information state dynamics (15).

Lemma 7: For any $u \in \boldsymbol{U}, U: \boldsymbol{R}^{n} \rightarrow \boldsymbol{R}$, and $p_{k} \in \mathcal{E}$

$$
\sup _{x \in \boldsymbol{R}^{n}} Q_{U}^{p_{k}}(x, u) \geq \sup _{y \in \boldsymbol{R}^{t}}\left(H\left(p_{k}, u, y\right), U\right) .
$$

This immediately yields a sufficient condition for the certainty equivalence controller to be a solution of the output feedback problem. 

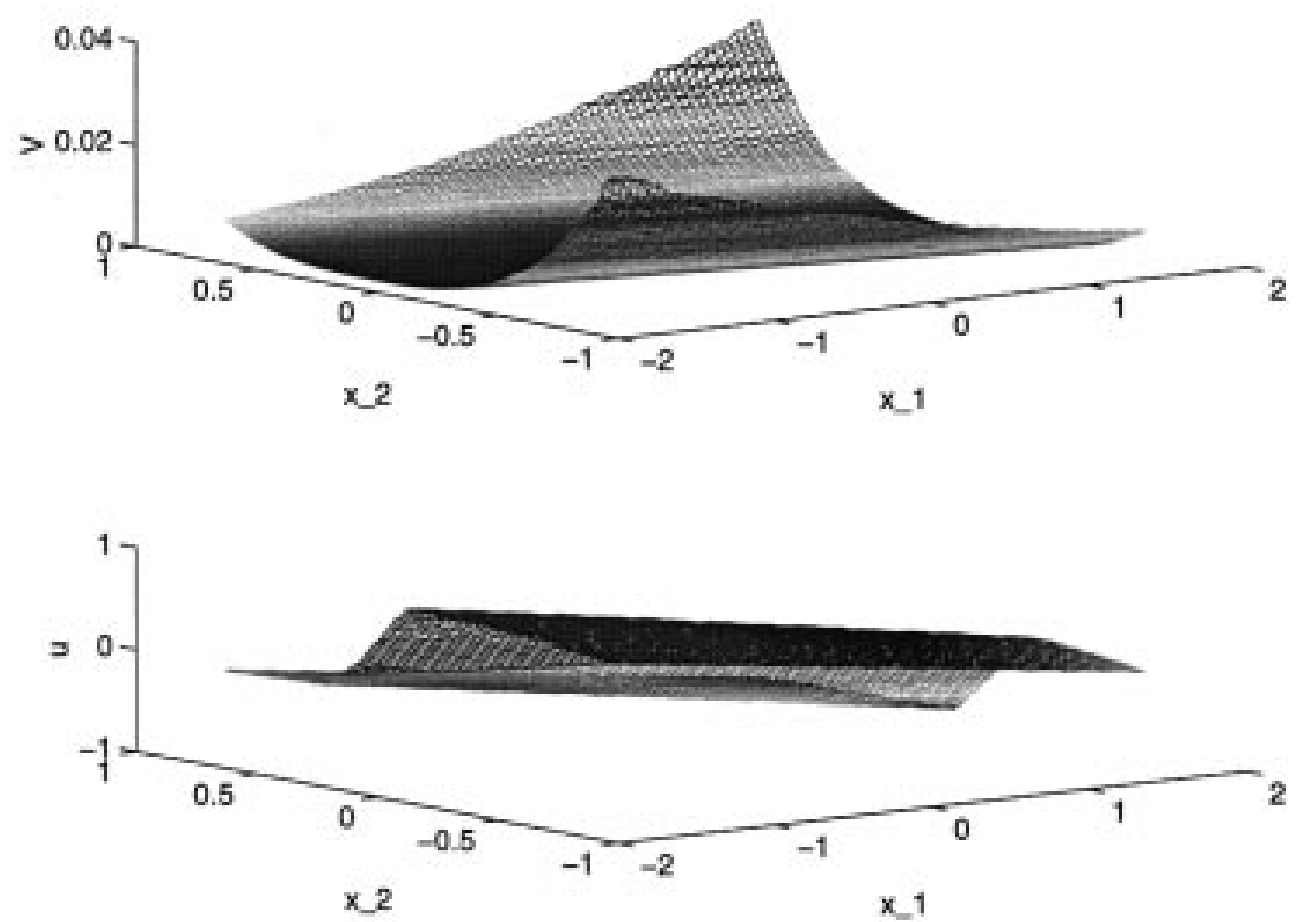

Fig. 2. Solution to the state feedback dynamic programming equation $V$ (top) and the control policy $u$ (bottom) for $\gamma=0.145$.

Theorem 19: Assume that

$$
\left(p_{k}, V\right)=\inf _{u \in \boldsymbol{U}} \sup _{x \in \boldsymbol{R}^{2}} Q_{V}^{p_{k}}(x, u), \quad k=0,1, \cdots
$$

where $p_{k}$ is the information state trajectory generated by employing the certainty equivalence controller, then the certainty equivalence controller solves the output feedback robust control problem provided that $\left(p_{0}, V\right)=0$.

Proof: Clearly, the conditions of the theorem imply that

$$
\left(p_{k}, V\right)=\sup _{x \in \boldsymbol{R}^{n}} \inf _{u \in \boldsymbol{U}} Q_{V}^{p_{k}}(x, u)=\inf _{u \in \boldsymbol{U}} \sup _{x \in \boldsymbol{R}^{n}} Q_{V}^{p_{k}}(x, u)
$$

Hence, a saddle point exists, and for any $\hat{x} \in$ $\operatorname{argmax}_{x \in \boldsymbol{R}^{n}}\left\{p_{k}(x)+V(x)\right\}$, and $\hat{u}=u_{F}(\hat{x})$, we have

$$
\left(p_{k}, V\right) \geq \sup _{y \in \boldsymbol{R}^{t}}\left(H\left(p_{k}, \hat{u}, y\right), V\right)
$$

via Lemma 7. Hence, $M\left(p_{k}\right)=\left(p_{k}, V\right)$ is a storage function, and the certainty equivalence controller solves the output feedback robust control problem, provided $\left(p_{0}, V\right)=0$.

Remark 13: We could have also considered any controller obtained in the following fashion. For a given function $U: \boldsymbol{R}^{n} \rightarrow \boldsymbol{R}$, with $U \geq 0, U(0)=0$, consider the information state feedback policy $\hat{u}$ obtained by $\hat{u}(p) \in$ $\operatorname{argmin}_{u \in \boldsymbol{U}} \sup _{x \in \boldsymbol{R}^{n}} Q_{U}^{p}(x, u)$. Now let the information state system be initialized via $p_{0}$ such that $\left(p_{0}, U\right)=0$, and let $p_{k}$ be the information state trajectory generated under $\hat{u}$. Then, if

$$
\left(p_{k}, U\right) \geq \inf _{u \in \boldsymbol{U}} \sup _{x \in \boldsymbol{R}^{n}} Q_{U}^{p_{k}}(x, u), \quad \forall k \geq 0
$$

$\hat{u}$ solves the output feedback robust control problem.

\section{EXAMPLE}

In this section we present a simple example. The system we consider is given by

$$
\begin{aligned}
x_{1}[k+1] & =0.8 x_{1}[k]+c_{1}\left(x_{2}[k]\right)^{2}+c_{2} u[k]+r_{1}[k] \\
x_{2}[k+1] & =x_{2}[k]+0.1 x_{1}[k]+r_{2}[k] \\
x_{3}[k+1] & =0.9 x_{3}[k]+0.1 r_{3}[k] \\
y[k+1] & =c_{3} x_{2}[k]+x_{3}[k]
\end{aligned}
$$

where all we know about the coefficients $c_{1}, c_{2}$, and $c_{3}$ is that they have values in the intervals $[0.4,0.9],[3,4]$, and $[1,1.1]$, respectively. Also, $r_{1}, r_{2}$, and $r_{3}$ are disturbance inputs bounded in the intervals $[-0.2,0.2],[-0.02,0.02]$, and $[-0.1,0.1]$, respectively. The state $x_{3}$ is employed to augment the system in order to reflect that the disturbance at the output is primarily of low frequency. Furthermore, $\boldsymbol{U}=[-2,2]$ and $X_{0}=[-0.75,0.75] \times[-0.6,0.6] \times[-0.1,0.1]$. The regulated output is chosen to be

$$
z_{k+1}=\left(x_{2}[k+1]\right)^{2} .
$$

Note that $l\left(x_{k+1}\right)=\left(x_{2}[k+1]\right)^{2}$ does not satisfy A5) directly. However, observe that the main role of $\mathcal{L}^{\gamma}$ is in showing that the states are ultimately bounded. From Corollary 6, it follows that if the problem is solvable for a given value of $\gamma$, then $\limsup _{k \rightarrow \infty} 2\left|x_{2}[k+1]\right| \leq \gamma$, and it follows that for this to be true, $x_{1}$ is ultimately bounded as well. Furthermore, it is clear that $x_{3}$ remains bounded.

In order to obtain a numerical solution, we discretize the state space as follows: $\Delta x_{1}=0.05, \Delta x_{2}=0.01$, and $\Delta x_{3}=0.01$. For this example, we employ $q=2$. We first solve the state feedback dynamic programming equation (11) using value iteration, employing a bisection search (as done 

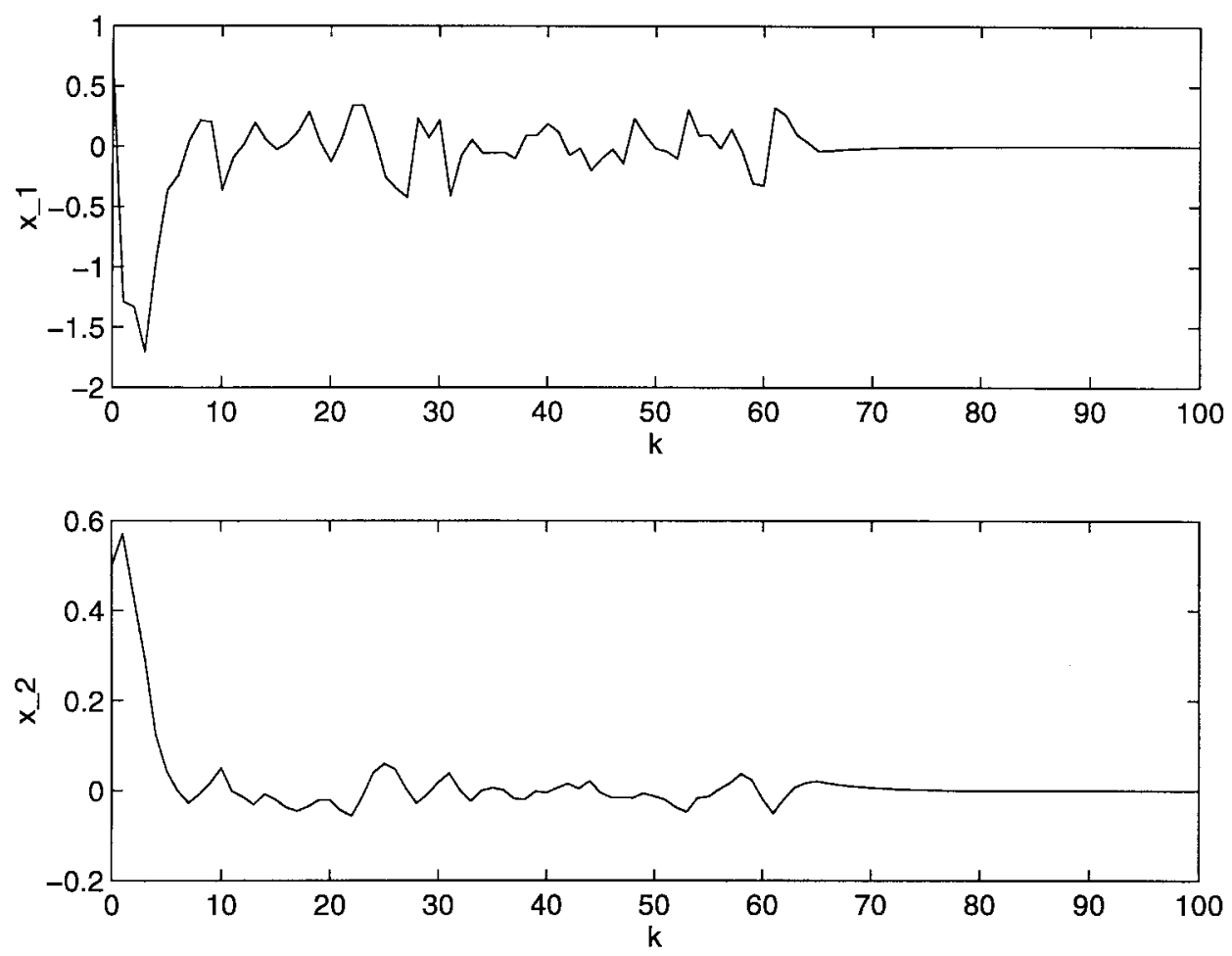

Fig. 3. State trajectories obtained employing state feedback $(\gamma=0.145)$.

for example in linear $H_{\infty}$ control) to obtain a suboptimal value for $\gamma$. For the state feedback case, we need only consider the dynamics associated with $x_{1}$ and $x_{2}$. We obtain $\gamma=0.145$ with the optimal $\gamma$ lying between 0.14 and 0.145 . The upper value function converges after 13 iterations. Fig. 2 shows the upper value function $(V)$ and the corresponding control policy $(u)$. Fig. 3 illustrates the trajectories obtained employing the state feedback control, starting the system with $x_{1}[0]=0.7$ and $x_{2}[0]=0.5$ and with $c_{1}, c_{2}, r_{1}$, and $r_{2}$ generated via uniform distributions to $k=60$, after which we set $c_{1}=$ $0.65, c_{2}=3.5, r_{1}=0$, and $r_{2}=0$.

For the output feedback case, we employ the certainty equivalence controller [obtained via (24)]. We first augment $V$ to include the third state $x_{3}$ by redefining $V$ as $V\left(x_{1}, x_{2}, x_{3}\right):=V\left(x_{1}, x_{2}\right)$, where the right-hand side is obtained by solving the state feedback problem. In order to check the conditions for certainty equivalence (Theorem 19) we resort to simulations, with the idea being to increase $\gamma$ (and recomputing $V$ ) from the suboptimal value for the state feedback problem until we observe

$$
\left(p_{k+1}, V\right) \leq\left(p_{k}, V\right) \leq \cdots \leq\left(p_{0}, V\right)
$$

consistently for repeated simulations at that value of $\gamma$. In each case, we initialize $p_{0}$ as $p_{0}(x)=-V(x)-10|x|^{2}$. We start with an initial value of $\gamma=0.15$ and increase it in increments of 0.025 . The first value of $\gamma$ for which the above inequalities are satisfied is $\gamma=0.475$. Fig. 4 shows the state trajectories $\left(x_{1}\right.$, and $\left.x_{2}\right)$ obtained by the certainty equivalence controller, along with the certainty equivalence estimates [i.e., $\hat{x}$ obtained in (24)], where all the disturbances are generated via uniform distributions to $k=50$, and the initial states were picked as $x_{1}[0]=0.5, x_{2}[0]=0.5$, and $x_{3}[0]=0$. After $k=50$, we set $c_{1}=0.65, c_{2}=3.5, c_{3}=1.05$, and $r_{1}=r_{2}=r_{3}=0$.

\section{APPENDIX}

We study the convergence of

$W_{k}^{\bar{u}}=\sup _{r \in \mathcal{F}\left(\bar{x}_{k}, \bar{u}_{k}\right)}\left(\left|l\left(r, \bar{u}_{k}\right)-l\left(\bar{x}_{k+1}, \bar{u}_{k}\right)\right|^{q}-\gamma^{q}\left|r-\bar{x}_{k+1}\right|^{q}\right)$

to zero, where $\bar{x}$ is a trajectory generated by the control $\bar{u}$.

Lemma 8: If $W_{k}^{\bar{u}} \rightarrow 0$, as $k \rightarrow \infty$, then $\forall \epsilon>0, \exists K$ such that $\forall k \geq K, \exists \delta$ such that

$$
\left|r-\bar{x}_{k+1}\right|<\delta, r \neq \bar{x}_{k+1} \Longrightarrow\left|l\left(\bar{x}_{k+1}, \bar{u}_{k}\right)-l\left(r, \bar{u}_{k}\right)\right|<\epsilon .
$$

Proof: Suppose the contrary, then $\exists \epsilon>0$ such that $\forall K, \exists k \geq K$, such that $\forall \delta>0$

$$
\left|r-\bar{x}_{k+1}\right|<\delta, r \neq \bar{x}_{k+1} \Longrightarrow\left|l\left(\bar{x}_{k+1}, \bar{u}_{k}\right)-l\left(r, \bar{u}_{k}\right)\right| \geq \epsilon .
$$

Fix $\delta$ such that $0<\delta<\epsilon^{1 / q}$. Then for any $s \in$ $B_{\delta / \gamma}\left(\bar{x}_{k+1}\right) \bigcap \mathcal{F}\left(\bar{x}_{k}, u_{k}\right) \subset \mathcal{F}\left(\bar{x}_{k}, u_{k}\right)$, with $s \neq \bar{x}_{k+1}$

$\left|l\left(\bar{x}_{k+1}, u_{k}\right)-l\left(s, \bar{u}_{k}\right)\right|^{q}-\gamma^{q}\left|\bar{x}_{k+1}-s\right|^{q} \geq \epsilon-\delta^{q}=\eta>0$.

This contradicts the convergence of $W_{k}^{\bar{u}}$.

Remark 14: The above lemma gives a necessary condition for the sequence $W_{k}^{\bar{u}}$ to converge.

Lemma 9: If $W_{k}^{\bar{u}} \rightarrow 0$ as $k \rightarrow \infty$, then $\forall \epsilon, \hat{\epsilon}>0, \exists K$ such that $\forall k \geq K, \exists r \in B_{\epsilon}\left(\bar{x}_{k+1}\right) \bigcap \mathcal{F}\left(\bar{x}_{k}, \bar{u}_{k}\right)$ with $r \neq \bar{x}_{k+1}$ and

$$
\frac{\left|l\left(r, \bar{u}_{k}\right)-l\left(\bar{x}_{k+1}, \bar{u}_{k}\right)\right|}{\left|r-\bar{x}_{k+1}\right|}<\gamma+\hat{\epsilon} .
$$



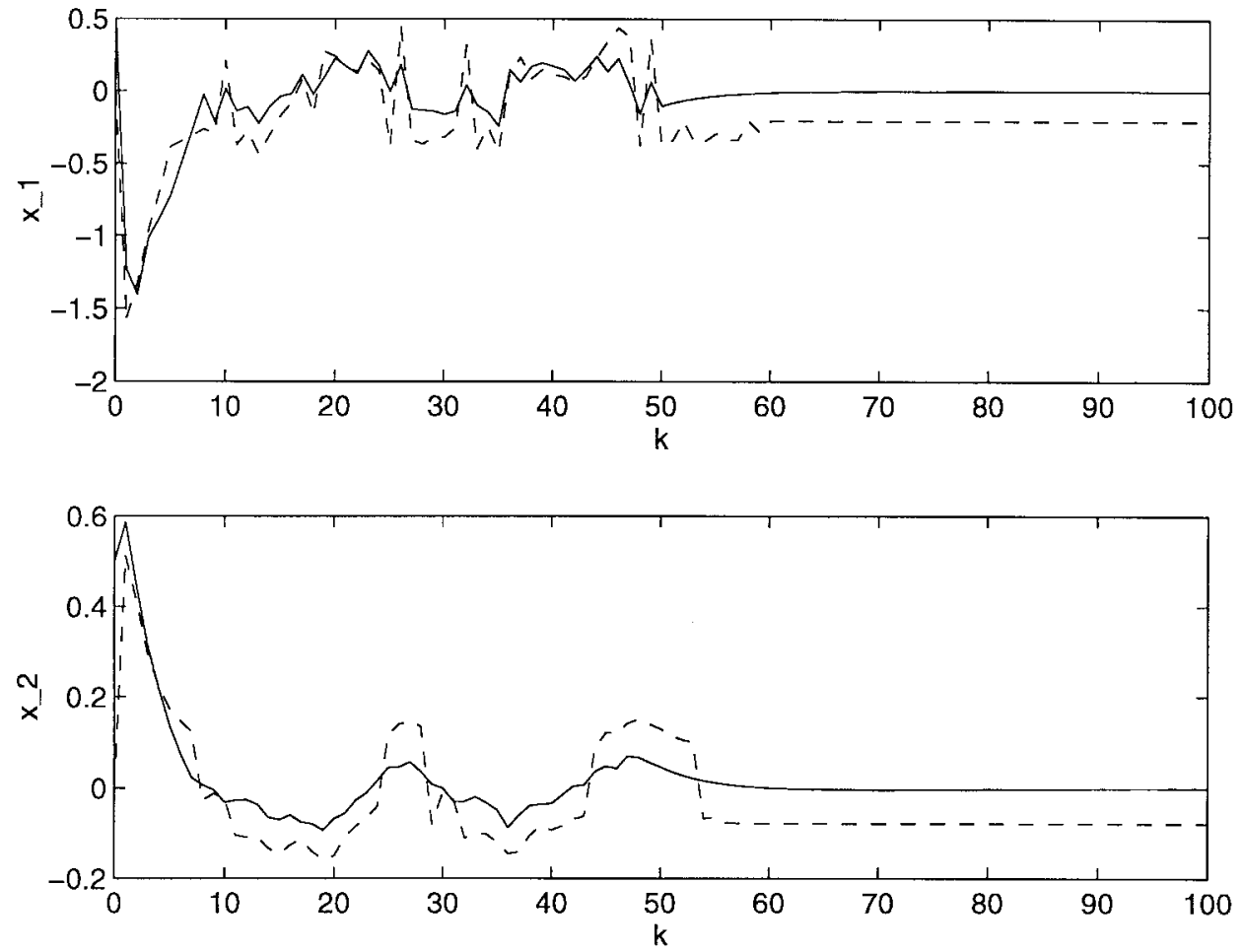

Fig. 4. States $x_{1}$ and $x_{2}$ (solid) along with their certainty equivalence estimates (dashed) $(\gamma=0.475)$.

Proof: We show the proof by contradiction. $\exists \hat{\epsilon}, \epsilon>0$, such that $\forall K, \exists k \geq K$ such that

$$
\begin{aligned}
& \frac{\left|l\left(r, \bar{u}_{k}\right)-l\left(\bar{x}_{k+1}, \bar{u}_{k}\right)\right|}{\left|r-\bar{x}_{k+1}\right|} \\
& \quad \geq \gamma+\hat{\epsilon}, \quad \forall r \in B_{\epsilon}\left(\bar{x}_{k+1}\right) \bigcap \mathcal{F}\left(\bar{x}_{k}, \bar{u}_{k}\right), \\
& r \neq \bar{x}_{k+1} .
\end{aligned}
$$

Hence, $\exists \eta>0$ such that

$$
\left|l\left(r, \bar{u}_{k}\right)-l\left(\bar{x}_{k+1}, \bar{u}_{k}\right)\right|^{q}-\gamma^{q}\left|r-\bar{x}_{k+1}\right|^{q} \geq \eta\left|r-\bar{x}_{k+1}\right|^{q} .
$$

Let $r \in B_{\epsilon}\left(\bar{x}_{k+1}\right) \cap \mathcal{F}\left(\bar{x}_{k}, \bar{u}_{k}\right)$ be such that $\epsilon>\mid r-$ $\bar{x}_{k+1} \mid>(\epsilon / 2)$. Thus

$$
\left|l\left(r, \bar{u}_{k}\right)-l\left(\bar{x}_{k+1}, \bar{u}_{k}\right)\right|^{q}-\gamma^{q}\left|r-\bar{x}_{k+1}\right|^{q} \geq \eta \frac{\epsilon^{q}}{2^{q}}=\hat{\eta}>0 .
$$

Hence, $\exists \hat{\eta}>0$ such that $\forall K, \exists k \geq K$ such that

$$
W_{k}^{\bar{u}} \geq \hat{\eta}
$$

Hence, we get a contradiction.

Corollary 6: If $W_{k}^{\bar{u}} \rightarrow 0$, then

$$
\limsup _{k \rightarrow \infty}\left|\frac{\partial}{\partial x} l\left(\bar{x}_{k+1}, \bar{u}_{k}\right)\right| \leq \gamma .
$$

Proof: Take the limit of (26) as $\epsilon, \hat{\epsilon} \rightarrow 0$, using A4) and A5).

\section{REFERENCES}

[1] B. R. Barmish, New Tools for Robustness of Linear Systems. New York: Macmillan, 1994

[2] K. Zhou and P. P. Khargonekar, "On the stabilization of uncertain linear systems via bound invariant Lyapunov functions," SIAM J. Contr. Optim., vol. 26, no. 6, pp. 1265-1273, 1988.

[3] I. R. Peterson, "Stability of an uncertain linear system in which uncertain parameters enter into the input matrix," SIAM J. Contr. Optim., vol. 26, no. 6 , pp. 1257-1264, 1988.

[4] I. R. Peterson and C. V. Hollot, "A Riccatti equation approach to the stabilization of uncertain linear systems," Automatica, vol. 22, no. 4, pp. 397-411, 1986.

[5] B. R. Barmish, I. R. Peterson, and A. Feuer, "Linear ultimate boundedness control of uncertain dynamical systems," Automatica, vol. 19, no. 5, pp. 523-532, 1983

[6] M. A. Dahleh and J. B. Pearson, " $l^{1}$-optimal feedback controllers for MIMO discrete-time systems," IEEE Trans. Automat. Contr., vol. 32, pp. 314-322, Apr. 1987.

[7] J. S. Shamma, "Construction of nonlinear feedback for $l_{1}$-optimal control," in Proc. 33rd IEEE Conf. Decision Contr., 1994, pp. 40-45.

[8] P. Peleties and R. DeCarlo, "Asymptotic stability of m-switched systems using Lyapunov-like functions," in Proc. Amer. Contr. Conf., 1991, pp. $1679-1684$.

[9] A. Gollu and P. Varaiya, "Hybrid dynamical systems," in Proc. 28th IEEE Conf. Decision Contr., 1989, pp. 2708-2712.

[10] M. S. Branicky, "Stability of switched and hybrid systems," in Proc. 33rd IEEE Conf. Decision Contr., 1994, pp. 3498-3503.

[11] A. F. Filippov, Differential Equations with Discontinuous Righthand Sides. New York: Kluwer, 1988.

[12] W.-M. Lu, "Attenuation of persistent $L_{\infty}$-bounded disturbances for nonlinear systems," in Proc. 34th IEEE Conf. Decision Contr., 1995, pp. 829-834.

[13] J.-P. Aubin, Viability Theory. Boston, MA: Birkhauser, 1991.

[14] M. R. James and J. S. Baras, "Robust $H_{\infty}$ output feedback control for nonlinear systems," IEEE Trans. Automat. Contr., vol. 40, pp. 1007-1017, June 1995.

[15] J. S. Baras and N. S. Patel, "Information state for robust control of set-valued discrete time systems," in Proc. IEEE Conf. Decision Contr., 1995, pp. 2302-2307.

[16] J. A. Ball and J. W. Helton, "Nonlinear $H_{\infty}$ control theory for stable plants," Math. Contr. Sig. Syst., vol. 5, pp. 233-261, 1992.

[17] J. Imura, T. Sugie, and T. Yoshikawa, "Bounded real lemma of nonlinear 
systems and its application to $H_{\infty}$ state feedback control," in Proc. 32nd IEEE Conf. Decision Contr., 1993, pp. 190-195.

[18] A. Isidori, "Dissipation inequalities in nonlinear $H_{\infty}$-control," in Proc. 31st IEEE Conf. Decision Contr., 1992, pp. 3265-3270.

[19] I. R. Peterson, B. D. O. Anderson, and E. A. Joncheere, "A first principles solution to the nonsingular $H_{\infty}$ control problem," Int. J. Robust Nonlinear Contr., vol. 1, pp. 171-185, 1991.

[20] A. J. van der Schaft, " $L_{2}$ gain analysis of nonlinear systems and nonlinear state feedback $H_{\infty}$ control," IEEE Trans. Automat. Contr., vol. 37, pp. 770-784, June 1992.

[21] J.-P. Aubin and I. Ekeland, Applied Nonlinear Analysis. New York: Wiley, 1984.

[22] J.-P. Aubin and H. Frankowska, Set-Valued Analysis. Boston, MA: Birkhauser, 1990.

[23] F. Blanchini, "Ultimate boundedness control for uncertain discrete-time systems via set-induced Lyapunov functions," IEEE Trans. Automat. Contr., vol. 39, pp. 428-433, Feb. 1994.

[24] F. Blanchini and M. Sznaier, "Persistent disturbance rejection via static state feedback," in Proc. 33rd IEEE Conf. Decision Contr., 1994, pp. 3159-3164.

[25] C. Castaing and M. Valadier, Convex Analysis and Measurable Multifunctions. New York: Springer-Verlag, 1977.

[26] P. Whittle, "Risk-sensitive linear quadratic gaussian control," Adv. Appl. Prob., vol. 13, pp. 764-777, 1981.

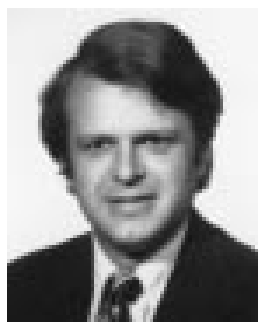

John S. Baras (S'73-M'73-SM'83-F'84) was born in Piraeus, Greece, on March 13, 1948. He received the B.S. degree in electrical engineering with highest distinction from the National Technical University of Athens, Greece, in 1970. He received the M.S. and Ph.D. degrees in applied mathematics from Harvard University, Cambridge, MA, in 1971 and 1973 respectively.

Since 1973 he has been with the Department of Electrical Engineering, University of Maryland, College Park, where he is currently a Professor and Member of the Applied Mathematics Faculty. From 1985 to 1991 he was the Founding Director of the Systems Research Center, now the Institute for Systems Research. In February of 1990 he was appointed to the Lockheed Martin Chair in Systems Engineering. Since 1991 he has been the Director of the Center for Satellite and Hybrid Communication Networks, a NASA Center for the Commercial Development of Space, which he cofounded. He has held visiting research scholar positions with Stanford, MIT, Harvard University, the Institute National de Reserche en Informatique et en Automatique, and the University of California, Berkeley. He has numerous publications in control and communication systems and is the coeditor of Recent Progress in Stochastic Calculus (New York: Springer-Verlag, 1990). His current research interests include stochastic systems and signal processing and understanding with emphasis on speech and image signals, real-time architectures, symbolic computation, intelligent control systems, robust nonlinear control, distributed parameter systems, hybrid communication network simulation, and management.

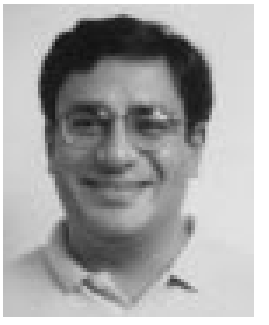

Nital S. Patel (S'91-M'96) received the B.Tech. degree from the Indian Institute of Technology, Kanpur, India, in 1991 and the M.S. and Ph.D. degrees from the University of Maryland, College Park, in 1993 and 1995, respectively, all in electrical engineering.

Currently, he is a Research Associate at the Institute for Systems Research. His research interests include robust nonlinear control, intelligent control, modeling, numerical methods for large-scale stochastic control problems, and their applications to control and coordination of manufacturing processes. 